\title{
RDL: A Language for Framework Instantiation Representation
}

Toacy C. Oliveira, Paulo S. C. Alencar, Carlos J.P. de Lucena, Donald D. Cowan.

\begin{abstract}
Reusing software artifacts for system development is showing increasing promise as an approach to reducing the time and effort involved in building new systems, and to improving the software development process and the quality of its outcome. However, software reuse has an associated steep learning curve, since practitioners must become familiar with a third party rationale for representing and implementing reusable assets. For this reason, enabling a systematic approach to the reuse process by making software reuse tasks explicit, allowing software frameworks to be instantiated using predefined primitive and complex reuse operations, and supporting the reuse process in a (semi-)automated way become crucial goals. In this paper, we introduce a systematic reuse approach and Reuse Description Language (RDL), a language designed to specify object-oriented framework instantiation processes, and an RDL execution environment, which is the tool support for definition and execution of reuse processes and framework

\footnotetext{
- Toacy C. Oliveira is with Faculty of Informatics at the Pontifical University Catholic of Rio Grande do Sul, Brazil . His e-mail address is toacy@inf.pucrs.br. Paulo S. C. Alencar and Donald D. Cowan are with the School of Computer Science at the University of Waterloo, Ontario, Canada. Their e-mail addresses are \{palencar, dcowan\}@csg.uwaterloo.ca respectively. Carlos J. P. de Lucena is with the Computer Science Department at the Pontifical University of Rio de Janeiro Brazil. His e-mail is lucena@inf.puc-rio.br.
} 
instantiations that lead to domain-specific applications. We illustrate our approach using JUnit, a framework for testing.

\section{Introduction}

Reusing software artifacts for system development has increasingly become a promising approach with the potential of reducing the time and effort involved in building new systems, and improving the software development process and the quality of its outcome. However, software reuse has an associated steep learning curve since practitioners must become familiar with a third party rationale for representing and implementing reusable assets [1]. As a result, the effort to reuse can be compared to the effort of developing a new artifact where a systematic approach is not adopted [2]. For this reason, the reuse process can be facilitated by representing reuse activities by means of process specifications that make explicit the software reuse tasks and thus liberating re-users from mining rationale directly from the reusable software asset design or code. Moreover, the process specifications can be mechanically processed enabling the construction of reuse wizards to automate or semi-automate the reuse process.

To mitigate the issues just raised, we have developed RDL, which stands for Reuse Description Language, a language that can be used procedurally for the process specification of object-oriented framework instantiation. When using RDL, framework developers can specify the sequence in which reuse actions must be executed as well as the constraints involved in the instantiation of reusable assets, such as frameworks. Moreover, RDL allows the specification of asynchronous reuse activities of the type widely used in Global Software Development [3]. As a result, the framework re-user is 
guided through a set of reuse actions and can focus on the relevant parts of the framework that should be adapted during the instantiation process.

In this paper, we thoroughly present the RDL language, depicting the elements RDL can manipulate, the syntax and semantics for the most important commands and the impact on design during the script execution. We also present RDL execution model, its associated software architecture and illustrate the approach applicability using the JUnit framework. In addition we briefly present the xFIT (XML-based Framework Instantiation Tool) approach, which is the reuse approach where the RDL execution environment is embedded in. It's worthwhile mentioning that RDL is part of a more comprehensive reuse approach used for Product Engineering [4] (see, for example, [5] [6] [7] [8]).

This paper is organized as follows. In Section 2 we describe related work on software reuse and explain how existing methods relate to the RDL approach. In Section 3 we provide background and motivation for this work. Section 4 introduces xFIT, a software reuse approach in which RDL in embedded. In Section 5 we briefly describe UML-FI, a representation for object-oriented frameworks and its flexible points, also called hotspots or points of variability. Section 6 describes RDL, the process language used to represent framework changes and instantiation processes, and provides its rationale and processoriented commands. In Section 7 we describe the underlying execution infrastructure for RDL, and in Section 8 we illustrate the applicability of RDL using JUnit, an objectoriented framework for testing. Finally, in Section 9 we present our conclusions and discuss some future directions. 


\section{Related Work}

In this section, we describe existing work on software reuse related to framework instantiation, product lines and model-driven architectures.

In [9] the authors have proposed a structured specification to support framework instantiation. They have introduced a template called Cookbook applied to a reuse document expressed in natural language, which can be difficult to follow because of the lack of a formal underlying approach. Moreover, Cookbooks can not be processed automatically, possibly leading to inconsistencies in their definition. As an extension to Cookbooks, Hooks [10] also provided a template for framework instantiation assistance and, as a result, share similar problems.

In the area of framework instantiation guidance, Active Cookbooks[11] advocate the use of Software Agents to execute instantiation plans. The main issue with the HiFi[11] approach is that it introduces non-standard notations such as TOON [11] into the application development process that can cause an extra burden to framework development. In OBS [12] the authors used a generative approach to framework instantiation that shares some of the features of our previous work [13]. However, the

OBS approach is based on ready-to-use blackbox frameworks, which restricts instantiation processes to component configuration, and does not focus on customization. 
Product Line Architectures also lead to approaches to support generating applications from pre-defined software components that can be configured based on a decision model[45]. In Gomaa [14] the author has presented a technique to develop Software Product Lines using UML diagrams and Feature Models. However, the lack of tool support causes difficulties for systematic adoption. Another initiative using the Features model is the Generative Programming (GP) approach described in [15], where the author proposes the development of Domain Specific Languages (DSLs that can be visual or textual) as a means to generate programs from high-level specifications and transformations. Although we share some underlying principles with the GP approach, we believe that well-known and standard notations and paradigms such as UML and object-oriented programming need to be used in the definition of process-related specifications and transformations. For this reason we use an extension of UML to represent frameworks and define $\mathrm{RDL}$ as a language to transform the extended UML models. In the same research direction, the WebML approach [16] guides developers through a set of pre-defined windows that can be seen as DSLs to collect applicationspecific information to create Web Applications. In the WebML approach the configuration knowledge is hard-coded into the tool, which complicates its extension to incorporate the evolution of specific domain requirements.

OMG's Model Driven Architecture [17] (MDA) is also an attempt to systematize reuse using UML, OCL [18] and software transformations [17]. The MDA approach can use modeling languages such as UML to represent software in a platform-independent manner. As a result, specific platform code can be automatically generated using a set of 
pre-defined transformations. However, MDA is still in the Request for Proposal (RFP) [19] phase wherein researchers are collaborating to develop a set of specifications, including notations for the transformation process.

\section{Background and Motivation}

We have developed object-oriented frameworks for several domains. Some of these frameworks were produced as result of academic research activities [20] [21] [22] [23], while others were developed in the context of partnerships between industry and academia $[24][25][26][27]$. Typically their development relied on gathering knowledge about a domain (Domain Analysis) and applying object-oriented programming expertise to implement flexible software [28]. However, the reuse processes were cumbersome since they were usually handled by programmers who had little experience in the design rationale for the frameworks. For instance, in the DSSFrame framework [26], where the extension points were constrained in a proper instantiation sequence, the framework designers had to spend a substantial amount of time to explain the design and supervise the team of "instantiators"".

In addition, as was the case with DSSFrame, reuse can be handled in an environment that is distributed, with developers in more than one site; or asynchronous where developers are not in constant collaboration because of different schedules. As a result, the sequence

\footnotetext{
${ }^{1}$ Those in charge of instantiation activities, who are also called re-users in this work.
} 
in which instantiation activities are executed is a major issue and must be supported by tools [29][3].

Under these circumstances, we began to develop approaches to assist instantiation processes [30]. However, the need for tools was very clear and, therefore, we concentrated on the specification and development of techniques for this purpose. The baseline specification we assumed for these tools had three key constraints:

$\Rightarrow$ Use standards found in the Software Engineering discipline such as UML, XMI [31] and typical object-oriented programming activities, to reduce the approach's learning curve.

$\Rightarrow$ Represent the process in a way that can be automated by an execution environment.

$\Rightarrow$ Assist the re-user as much as possible.

As a result, we developed xFIT, the XML-based Framework Instantiation Tool, which embodies our reuse approach in a tool. xFIT guides re-users through the framework instantiation process by exposing the frameworks' high-level characteristics through a Feature Model [32]. Such features are then traced to design elements specified in UMLFI, a UML profile responsible for exposing framework extension points at the design level. In order to automate the process, RDL expresses how each extension point should be extended to create the final application. In this approach XML is a key technology, since most of documents are expressed in XML.

\section{Introducing the xFIT Approach}

The xFIT approach is intended to facilitate the framework instantiation processes by means of a tool that executes instantiation scripts written in RDL. Ideally xFIT takes as 
input three types of documents as shown in Figure 1: a Features Model, an Annotated Framework Design, and a RDL Instantiation Script.

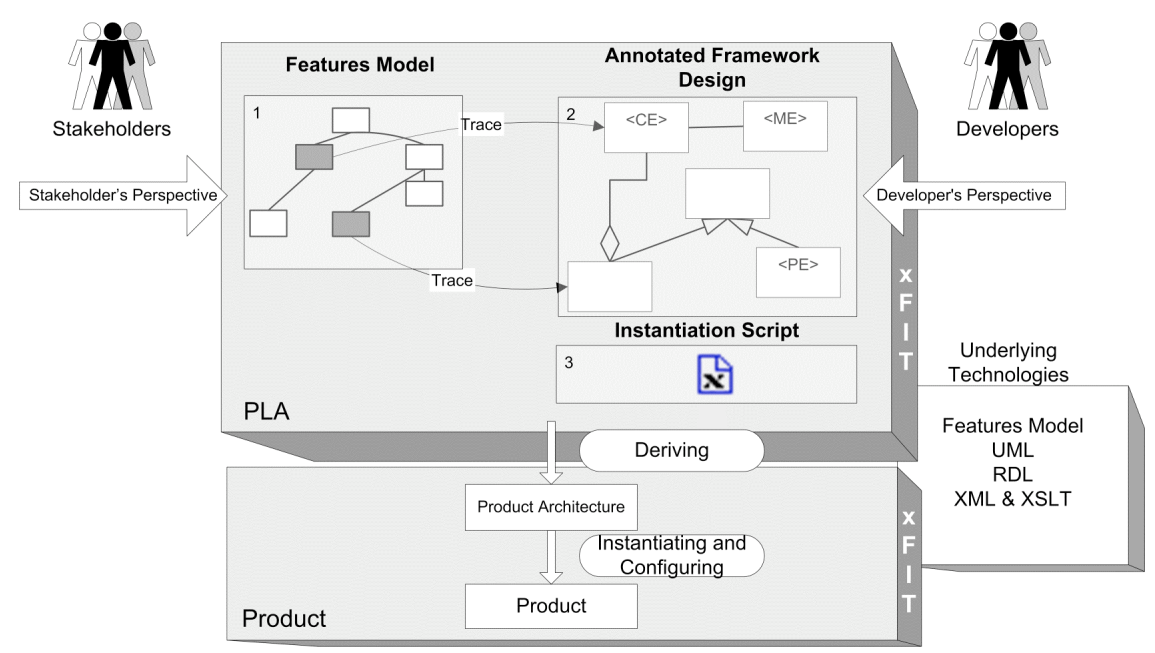

Figure 1 - An overview of our approach from [5].

The Features Model is used as the original formulation [32] and is intended to provide a high-level visualization for the framework's characteristics. The annotated framework design is the representation of an object-oriented framework using UML. Actually, in order to represent all flexible characteristics embedded in the framework design, we have developed and adopted UML-FI (UML for Framework Instantiation), which is an extended version of UML designed to emphasize the flexible elements in a OO design. UML-FI will be briefly introduced in Section 5.

The last document is the Instantiation Script, which specifies the instantiation process so that a framework re-user can be guided in accommodating the application specific needs 
into the framework's extension points. In order to achieve a succinct process description we have developed RDL (Reuse Description Language), a language that allows the specification of order and state dependencies among object-oriented programming activities that are often used when instantiating an incomplete design.

To integrate the three technologies, Features, a Framework's Annotated Design and the Instantiation Script, we created a mapping technique using UML trace mechanisms that relates the flexibility represented by optional and alternative features found in the Features Model, to extension points (hotspots) in the framework design [7]. By doing so, the xFIT execution environment can partially generate the RDL script that is used to tailor the framework design. We achieved this goal by creating RDL instantiation templates that are associated with each UML-FI stereotype (see Section 5) and executed when a corresponding feature was chosen.

We also have the ability to run RDL scripts that are manually created by the Framework Designer and are not related to a Features Model. We believe that for frameworks with several extension points, where each feature is not easily related to a single design element such as a class, the mapping approach may not be feasible.

Our approach consists of two different phases: the Framework Engineering Phase and the Framework Instantiation Phase. During the Framework Engineering Phase, the framework designer (or engineer) needs to develop the framework and provide the mechanisms to facilitate its reuse (Figure 2). In other words the framework design must 
be annotated using UML-FI stereotypes and tagged values and a Features Model must be created to represent the framework's characteristics at a high-level of abstraction. This Features Model will also expose aspects of the framework's flexibility and, finally, relate features and UML-FI design elements. It is possible to skip the last phase and to create the RDL scripts manually.

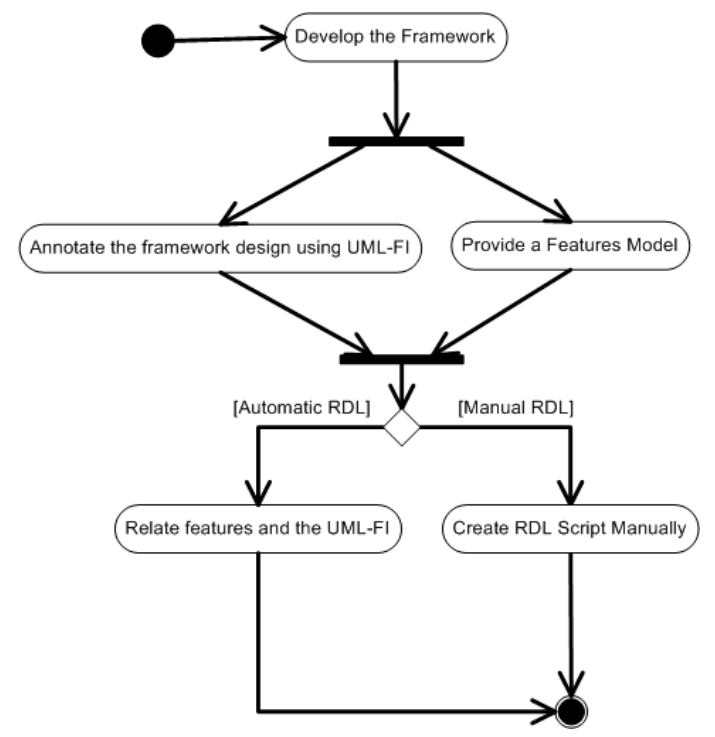

Figure 2 - Framework engineering process.

The Framework Instantiation Time is when a framework's extension points are completed with application-specific increments [28]. In this context, the framework instantiation process, which shows how to produce the software product (the application), begins with the stakeholder ${ }^{2}$ selecting the features to be included in the specific software

\footnotetext{
${ }^{2}$ Stakeholder in our scenario is someone interested in the framework reuse.
} 
product. The selected characteristics (shown in dark gray in the Features Model of Figure 1), together with the annotated class model, will be provided as input into the xFIT tool that will generate an instantiation script containing the necessary steps to instantiate an application with the desired characteristics.

The proposed instantiation process finishes when the instantiation script is executed using an RDL execution environment such as xFIT (Figure 3c). The RDL execution environment will then modify the original design of a framework by inserting/removing some design elements to adapt the hotspots to meet the requirements of a specific application. It is worth mentioning that this last step is not entirely automatic in that the application developers need to provide additional information such as class names, attribute names and types, and method names.

Figure 3 illustrates the RDL execution scenario. Figure 3a represents a fragment of a framework design in UML containing a class Figure, which is an extension point, and the associated RDL script. The Instantiation occurs when the application increments represented by the classes Circle and Square (Figure 3b) are combined with the framework design. 


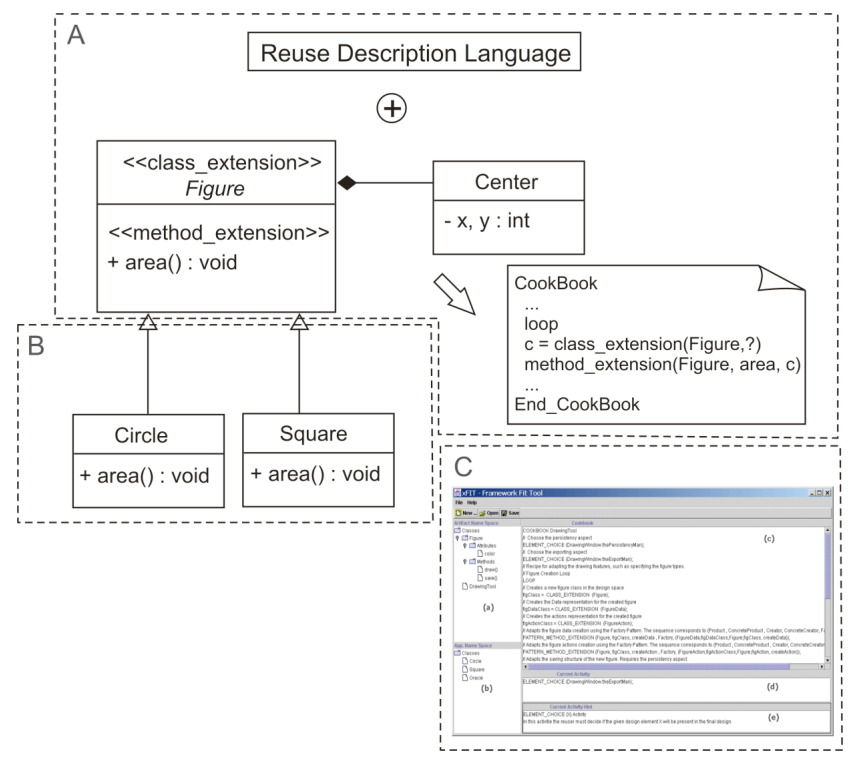

Figure 3 RDL Execution approach

Although the best way to follow an RDL script is using an execution environment such as xFIT, we believe that RDL commands can be processed by humans. We base this assumption on the fact that RDL syntax and semantics are based on ordinary programming activities thus facilitating understanding.

We close this overview with an important additional remark: the documents used in our approach are XML-based. We have adopted the XML technology, since it has broad support from standards bodies such as $\mathrm{W} 3 \mathrm{C}$ and it is commonly accepted or adopted by the academic community and industry. Moreover, based on our past experience [33][13], XML models are suitable for program manipulation and can be translated to formal models that can be validated. 


\section{UML-FI}

In this section we present the main UML-FI (UML for Framework Instantiation) notation constructs, an extension we have developed to specify object-oriented frameworks and the instantiation of their extension points. UML-FI is a UML profile containing stereotypes and tagged-values (i.e., UML extension mechanisms) to indicate, at the design level, the object-oriented activities that should be performed when instantiating a framework.

\begin{tabular}{|l|l|l|}
\hline Notation & Base Class & Description \\
\hline$<<$ CLASS_EXTENSION $>>$ & class & Indicates that the current class requires subclassing. \\
\hline $\begin{array}{l}<\text { PATTERN_CLASS_ } \\
\text { EXTENSION }>\end{array}$ & class & $\begin{array}{l}\text { Indicates that the current class requires subclassing, which is } \\
\text { achieved through the application of a pattern. }\end{array}$ \\
\hline$<<$ SELECT_CLASS_EXTENSION $>>$ & class & $\begin{array}{l}\text { Indicates that the current class requires subclassing, which is } \\
\text { achieved by selecting one of its known subclasses. }\end{array}$ \\
\hline$<<$ METHOD_EXTENSION $>>$ & method & $\begin{array}{l}\text { Indicates that the current method requires redefinition in a } \\
\text { subclass. }\end{array}$ \\
\hline$<<$ PATTERN_METHOD_EXTENSION $>>$ & method & $\begin{array}{l}\text { Indicates that the current method requires redefinition in a } \\
\text { subclass, which is achieved with the application of a pattern. }\end{array}$ \\
\hline$<<$ VALUE_ASSIGNMENT_EXTENSION $>>$ & attribute & Indicates that the current attribute requires a value. \\
\hline$<<$ VALUE_SELECTION_EXTENSION $>>$ & attribute & $\begin{array}{l}\text { Indicates that the current attribute requires a value, which can } \\
\text { selected from a list of given values. }\end{array}$ \\
\hline
\end{tabular}

Table 1 - UML-FI Stereotypes.

\begin{tabular}{|l|l|l|}
\hline Tag Name & Stereotype & Description \\
\hline Presence & Class, Attribute, Method & $\begin{array}{l}\text { Qualifies the design element as mandatory in the final design. The default } \\
\text { is "presence = mandatory". } \\
\text { \{mandatory, optional }\end{array}$ \\
\hline
\end{tabular}

Table 2 - UML-FI Tagged values.

It is possible to indicate in UML-FI most basic object-oriented programming activities such as class extension, method redefinition and value assignment (useful for Blackbox framework instantiation [1]). These activities are defined by means of annotations (stereotypes and tagged values shown in Tables 1 and 2) in class diagrams (Figure 4). 


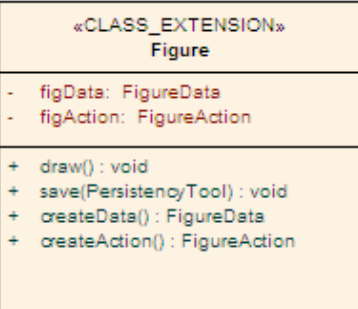

Figure 4 - Class extension stereotype.

In addition, extension points can also be mandatory or optional. Therefore, the re-user can decide if the associated design element will (or will not) be present in the final application. UML-FI indicates optional and mandatory aspects as a tagged-value named presence that can assume the values OPTIONAL or MANDATORY (Figure 5).

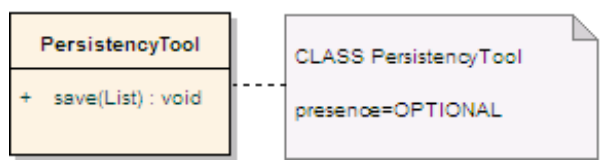

Figure 5 - Optional representation.

\section{RDL}

Instantiation processes tailor a framework's design and code based on carefully developed elements called Extension Points ${ }^{3}$ (EP). In order to adapt a framework's EP, a re-user must execute a set of Instantiation Tasks (IT) that are closely related to ordinary activities performed when programming. For instance, a whitebox framework's ITs can be related to specializing classes and/or redefining methods; on the other hand, a blackbox framework's ITs are focused on parameterization. Thus a language built to

\footnotetext{
${ }^{3}$ In this work the terms Extension Points and Hotspots are equivalent.
} 
specify a process to control the execution of ITs must use the same rationale adopted when programming the framework.

In order to systematize instantiation processes RDL allows the definition of an instantiation script document, containing the ITs required to derive the application from the framework's design and code. Moreover, ITs must be executed in a proper sequence, which can also be specified within a RDL script.

The structure of an RDL instantiation script is the same as found in most imperative programming languages, namely program constructs and procedures. However, in order to follow the usual framework instantiation terminology, we use the term Cookbook [9] to represent a program and Recipe [9] to represent a procedure. Every Cookbook must have a Recipe called main that identifies the instantiation process execution entry point as shown in Code 1.

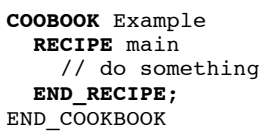

Code 1 - A Cookbook example.

Note that the instantiation process is totally dependent on the final application domain that constrains the extension points to a proper set of values. For instance, in an eCommerce framework, the elements can be books, CDs or cars and are not known during the framework engineering but only during instantiation. As a result, the RDL instantiation script does not have all the information needed to transform the framework design automatically into the application design. Instead, it must interrogate the re-user 
for the missing information. That is why we refer to our instantiation approach as being systematic, not automatic.

\subsection{Manipulated Elements}

The instantiation process must produce an application from an incomplete model, since extension points represent flexible characteristics in the model that can not be specified during framework design. For instance, a designer developing a framework for the eCommerce domain must not fix the element under trade, thus providing more reuse opportunities. ${ }^{4}$ Therefore, during instantiation of the eCommerce framework, new design elements to specify the product under trade must be added to bring the model to a complete and coherent state.

In our case, a model is a slight adaptation of UML Class Diagrams (see UML-FI in Section 5) and what RDL does is provide syntax and semantics to a process that manipulates meta-elements such as the ones present in the UML meta-model Abstract Syntax [34]. We did not use high level elements found in the meta-model hierarchy such as ModelElement, Feature or Classifier, instead we used specialized elements such as Class, Method, Attribute and Relationship, in order to reduce the number of elements RDL needs to manipulate and improve clarity. Moreover, using those specialized elements led to a straightforward connection with ordinary object-oriented programming principles.

\footnotetext{
${ }^{4}$ According to Parnas [35], the amount of reuse opportunities increases when you do not restrict the representation of an application family to a specific domain.
} 
It is important to mention that the meta-class ReusableElement was introduced in the UML meta-model as a super class for Class, Method and Attribute, and is manipulated by RDL scripts. In fact, some operation and attributes are common to all design elements, and using inheritance to capture the common features is an obvious choice.

Although UML focuses on design, code is also an important element in the reuse process. For that reason we decided to incorporate the representation of Programming Language idioms using the UML element named ProcedureExpression [34]. When an RDL script uses a ProcedureExpression, it becomes tied to that language and can not be used in instantiation processes with different languages. However, a framework is usually the incomplete version of a running application, being actually represented in a programming language that has already been chosen.

Another UML element that is manipulated with RDL is a Note. Notes allow framework designers to add extra documentation and instantiation hints. For example, assigning values to the parameters found in Blackbox Frameworks can be done anywhere in the code so there is no way to determine where such assignment will take place during the instantiation process. Sticking a note to a parameter with some documentation stating that the assignment must occur and listing the elements involved provides a hint about the parameter instantiation to the framework re-user. 


\subsection{RDL Commands}

Commands in RDL must ultimately cover object-oriented programming activities and ordinary programming statements such as loop, assignment and conditional. Although our underlying principle to create RDL was the same adopted by Meyer [36], who states that a language must be concise with few and seamless commands, our goal was also to raise RDL reuse expressiveness. To achieve this goal, we have incorporated a set of language elements specifically related to instantiation tasks and have organized RDL statements into five groups according to their purpose within the instantiation process: Basic Programming Tasks, Instantiation Specific Tasks, Pattern Specific Tasks, Sequence Specific Tasks and Miscellaneous Tasks (See Section 6.3).

The following sections 6.3 to 6.8 present RDL from its execution perspective, showing how design and code are changed after the execution of a command. Each Instantiation Task is presented in a table using the structure Command, Syntax, Description, Code and Design, where:

$\Rightarrow$ Command-Specifies the Command name to be used in a informal manner.

$\Rightarrow$ Syntax - Specifies the command sentence structure to be used within the RDL script. In some cases the notation used is similar to $\mathrm{C}$ functions as in as return_type CommandName (parameter_list), where return_type specifies the type of the returned element; CommandName is the name of the command; and parameter_list is a placeholder to receive arguments.

$\Rightarrow$ Description - Provides a textual description to the task intention.

$\Rightarrow$ Comments - Provides extra information when needed (this part is optional).

$\Rightarrow$ Code - Shows an example of use of the command within a RDL script.

$\Rightarrow$ Design - Illustrates how design is modified after the RDL script execution. 
In order to provide a more general and complete representation, a BNF-like specification is shown in Appendix I.

\subsection{Basic Programming Tasks}

Basic Programming Tasks (BPTs) represent ordinary design and code manipulation activities and are presented in Tables 3 to 7.

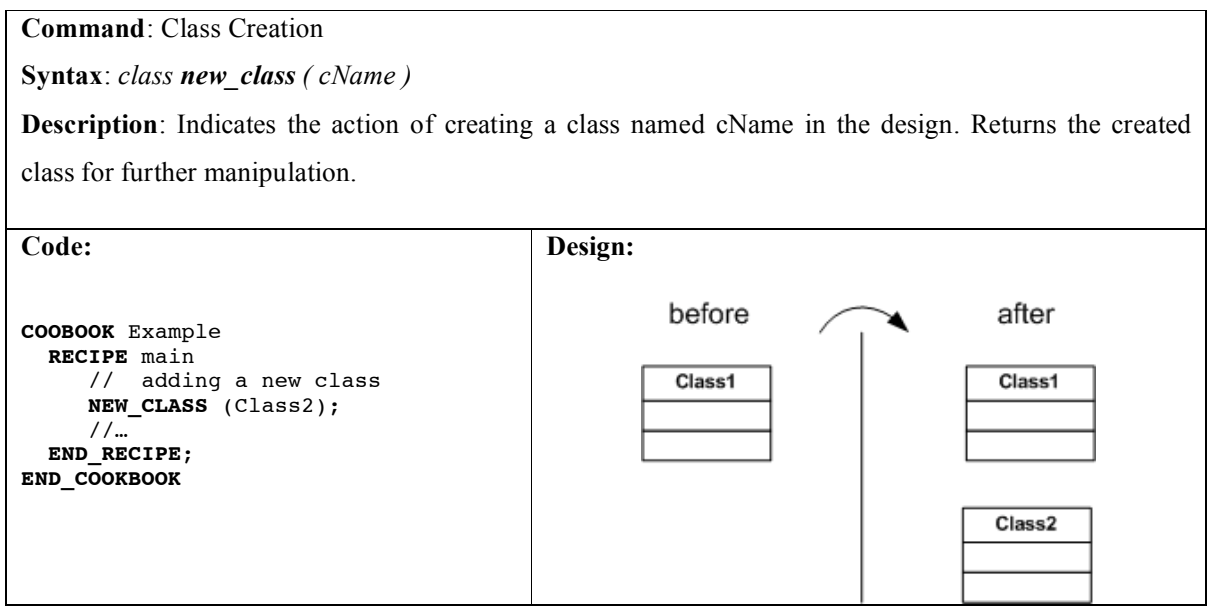

Table 3 - Class Creation Command

Command: Method Creation

Syntax: method new_method (c, metName)

Description: Indicates the action of creating a method named metName in class c. Returns the created method.

\begin{tabular}{|c|c|c|}
\hline Code: & Design: & \\
\hline $\begin{array}{l}\text { COOBOOK Example } \\
\text { RECIPE main }\end{array}$ & before & after \\
\hline $\begin{array}{l}\text { NEW_METHOD (Class1, operation } 1) \text {; } \\
\text { END_RECIPE; } \\
\text { END_COOKBOOK }\end{array}$ & & \begin{tabular}{|l|} 
\\
+operation10
\end{tabular} \\
\hline
\end{tabular}

Table 4 - Method Creation Command 


\begin{tabular}{|c|c|c|}
\hline \multicolumn{3}{|c|}{$\begin{array}{l}\text { Command: Attribute Creation } \\
\text { Syntax: attribute new_attribute (c, attName) } \\
\text { Description: Indicates the action of creating an attribute named attName in class c. Returns the created } \\
\text { attribute. }\end{array}$} \\
\hline $\begin{array}{l}\text { Code: } \\
\text { COOBOOK Example } \\
\text { RECIPE main } \\
\text { // adding a new attribute } \\
\text { NEW_ATTRIBUTE (Class1, attribute1); } \\
\quad / / \\
\text { END_RECIPE; } \\
\text { END_COOKBOOK }\end{array}$ & $\begin{array}{l}\text { Design: } \\
\text { before }\end{array}$ & after \\
\hline
\end{tabular}

Table 5 - Attribute Creation Command

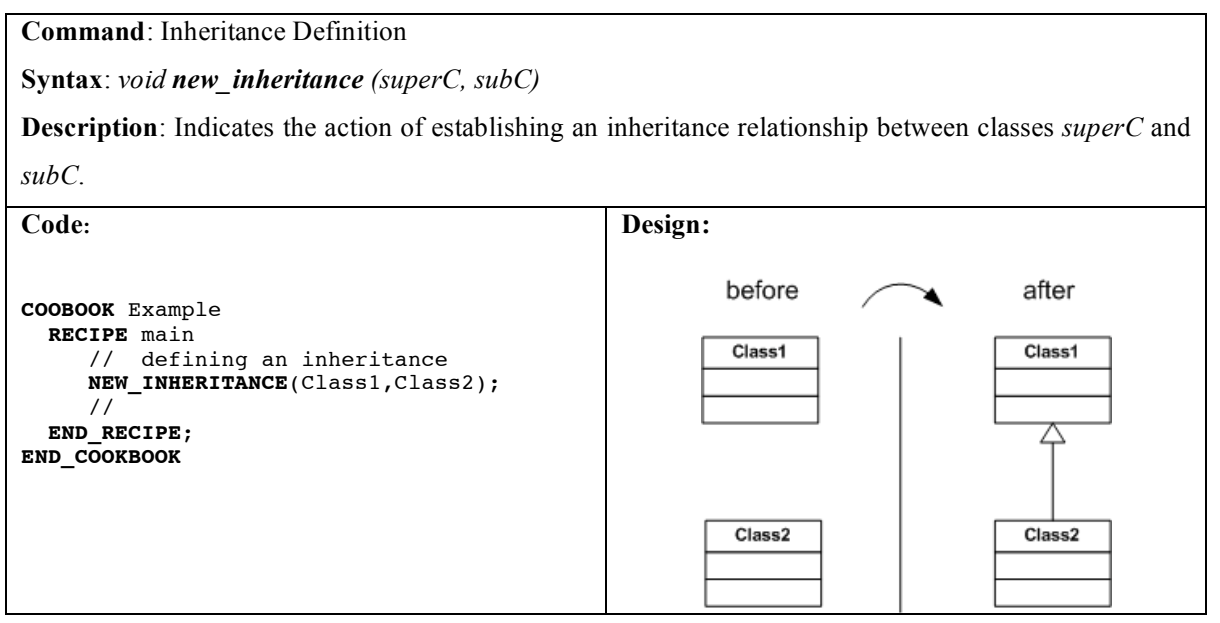

Table 6 - Inheritance Definition Command

Command: Code Assignment

Syntax: void add_code (class, method, code_string)

Description: Indicates the action of assigning a code to the given method.

Comments: In order to provide visual feedback a Note is also attached to the class parameter. 


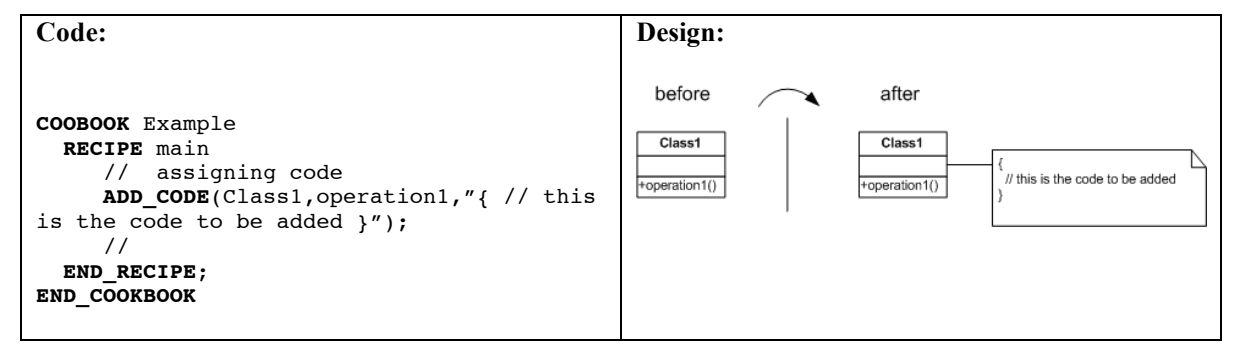

Table 7 - Code Assignment Command

\subsection{Instantiation Specific Tasks}

Instantiation Specific Tasks (ISTs) raise the abstraction level found in RDL scripts since they provide a vocabulary used when dealing with object oriented framework reuse. ISTs typically combine some basic tasks found in the Basic Programming Task group and they are specified in Tables 8 to 13 .

Command: Element Choice
Syntax: boolean element_choice (el)
Description: Indicates the action of choosing if the element $e l$ will be present in the final application
design. Returns true if the element was chosen; false otherwise.
Comments: This action depends on information only available at instantiation time, and thus it must
prompt the re-user for feedback. Note that the framework implementation must be carefully developed to
support the absence of the element to avoid compile time errors such as "element not found."
$\begin{aligned} & \text { Code: } \\ & \text { COOBOOK Example } \\ & \text { RECIPE main } \\ & \text { Choosing an element } \\ & \text { ELEMENT_CHOICE (C1.aComponent); } \\ & \text { I/ END_RECIPE; } \\ & \text { END_COOKBOOK }\end{aligned}$

Table 8 - Element Choice Command 


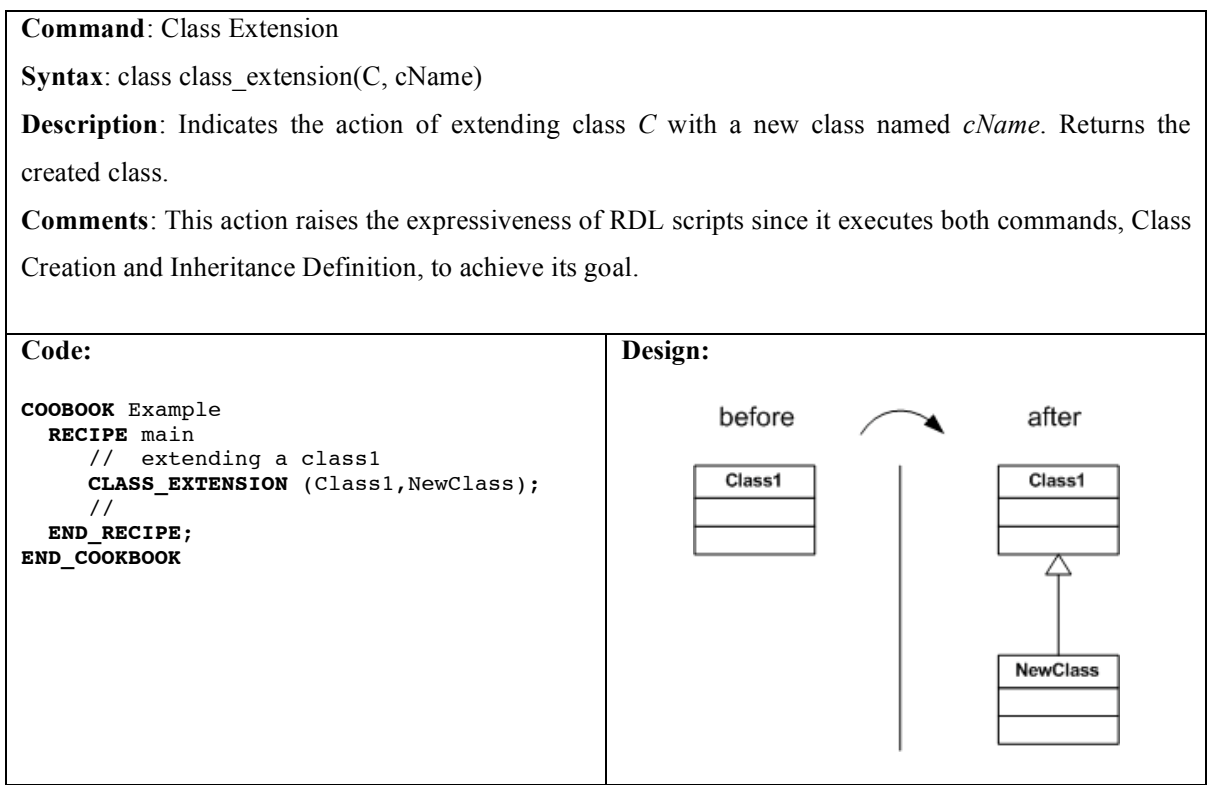

Table 9 - Class Extension Command

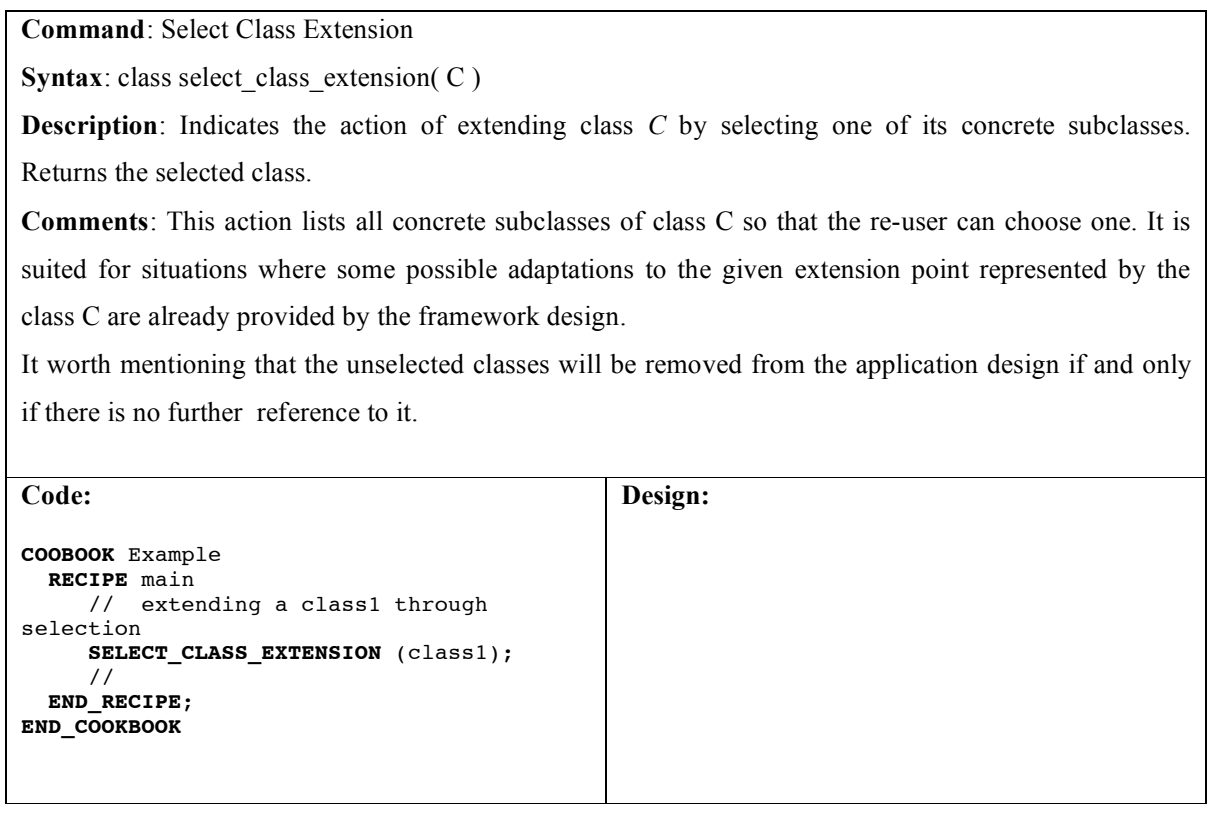




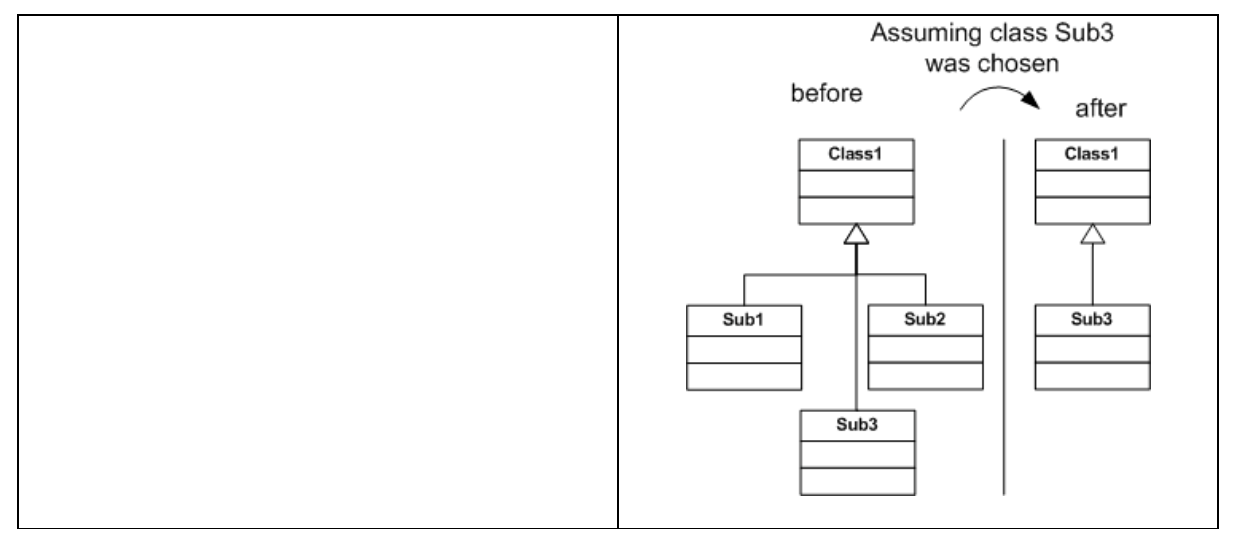

Table 10 - Select Class Extension Command

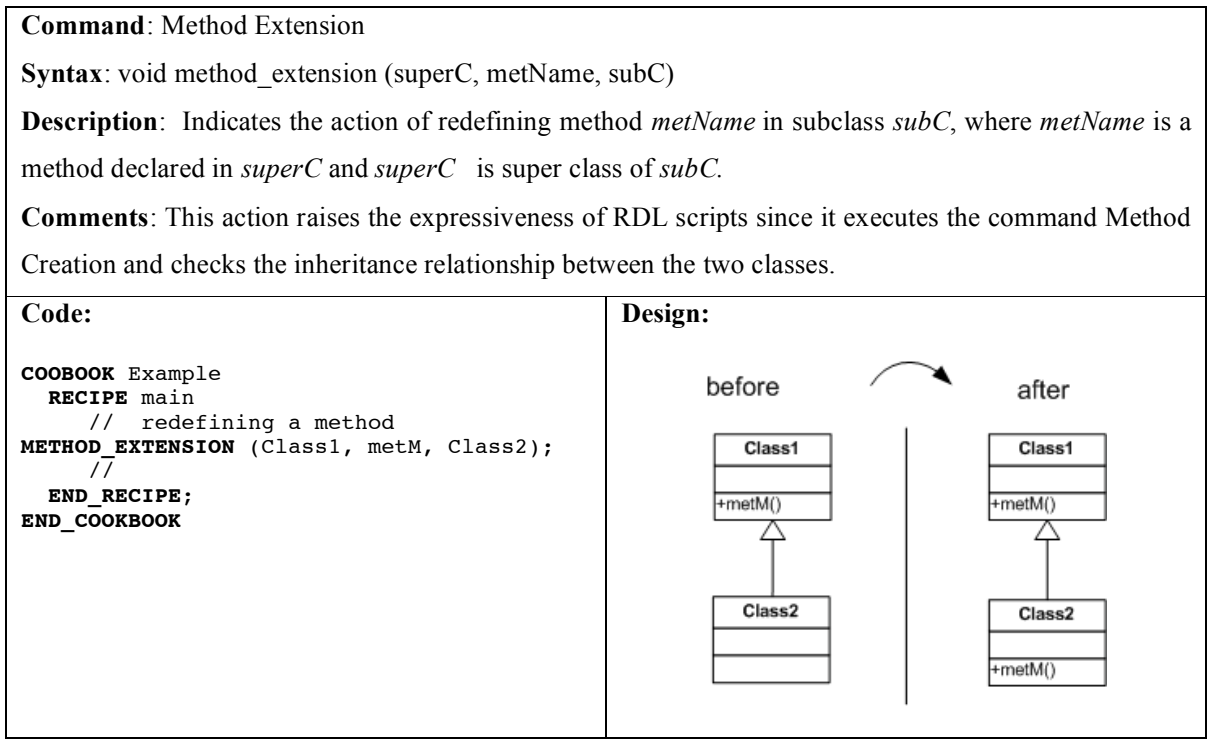

Table 11 - Method Extension Command

Command: Value Assignment

Syntax: void value_assigment (C, attName, V)

Description: Indicates the action of assigning the value $V$ to attribute attName of class $C$.

Comments: Since this action deals with code not design, the result of its execution is a note attached to class $C$. The syntax used in the note is the same as in C, i.e., attName $=\mathrm{V}$. 


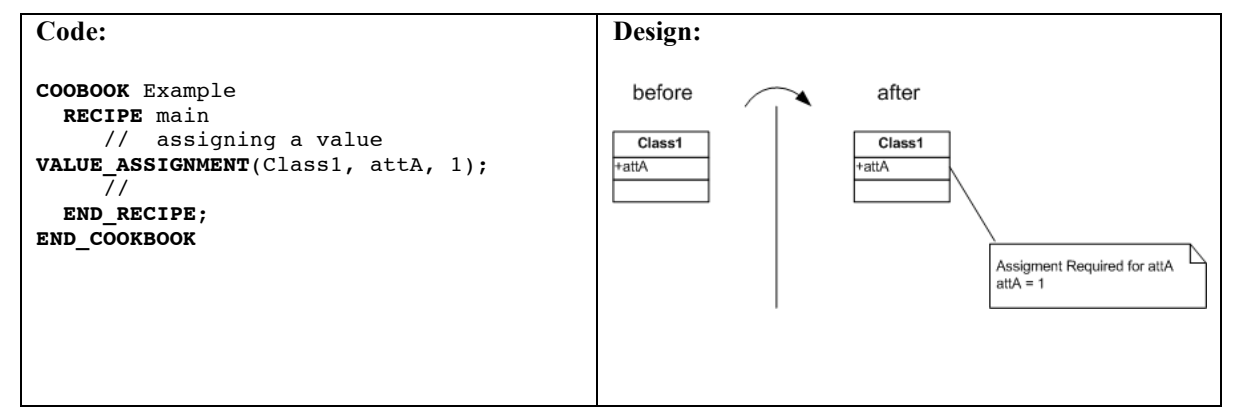

Table 12 - Value Assignment Command

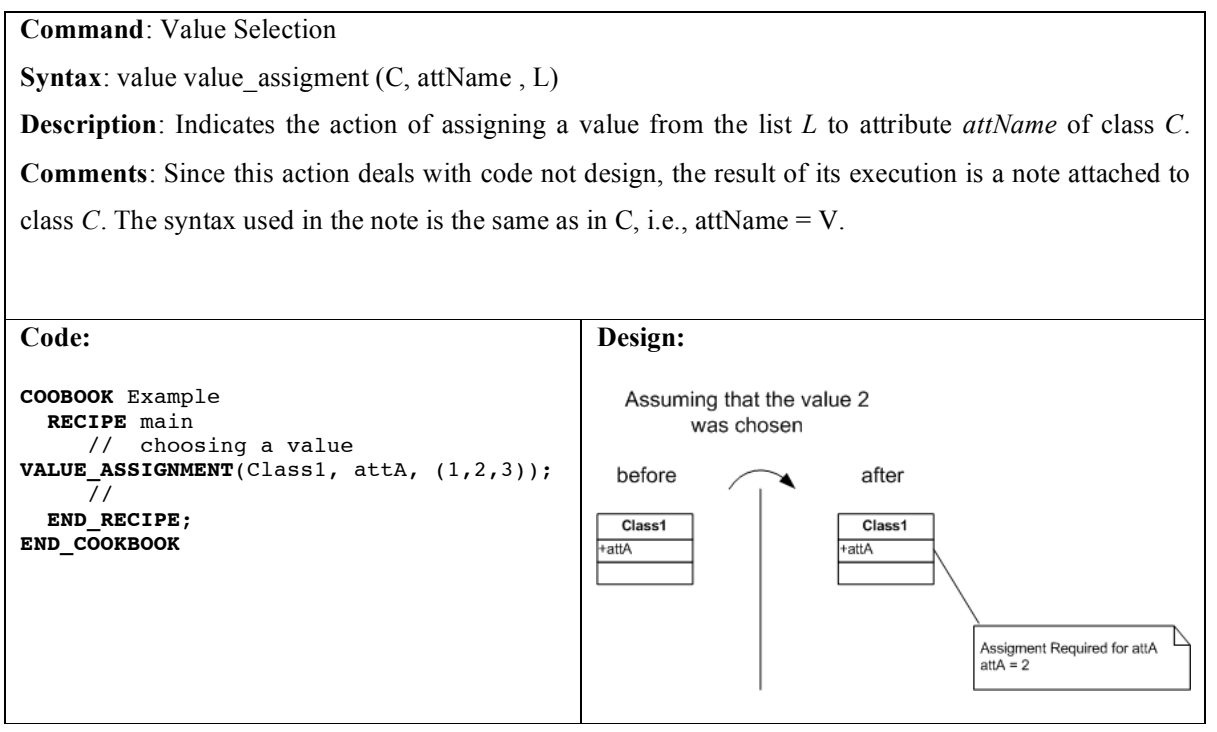

Table 13 - Value Selection Command

\subsection{Pattern Specific Tasks}

Pattern Specific Tasks support the assembly of several RDL commands into a special recipe, the Pattern Script, which is developed to render a sequence of instantiation actions that are relevant for the Framework's domain. For example, when instantiating the HotDraw [9] framework there must be a task related to the specification of drawing elements. As a result, for every drawing element that the HotDraw instance makes available in its palette, it is mandatory to provide its name, its icon and its associated 
drawing tool. To accomplish this goal several interconnected parts of HotDraw's design must be changed, and, for this reason, grouping such activities in a Pattern Script is recommended.

Another goal of Pattern Scripts is to promote reuse of RDL activities in recurrent instantiation processes by means of a name, in the same way as Design Patterns (that was the reason for introducing a Pattern name in the script). We illustrate the Pattern Specific Tasks in Tables 14 and 15 using some well-known Design Patterns.

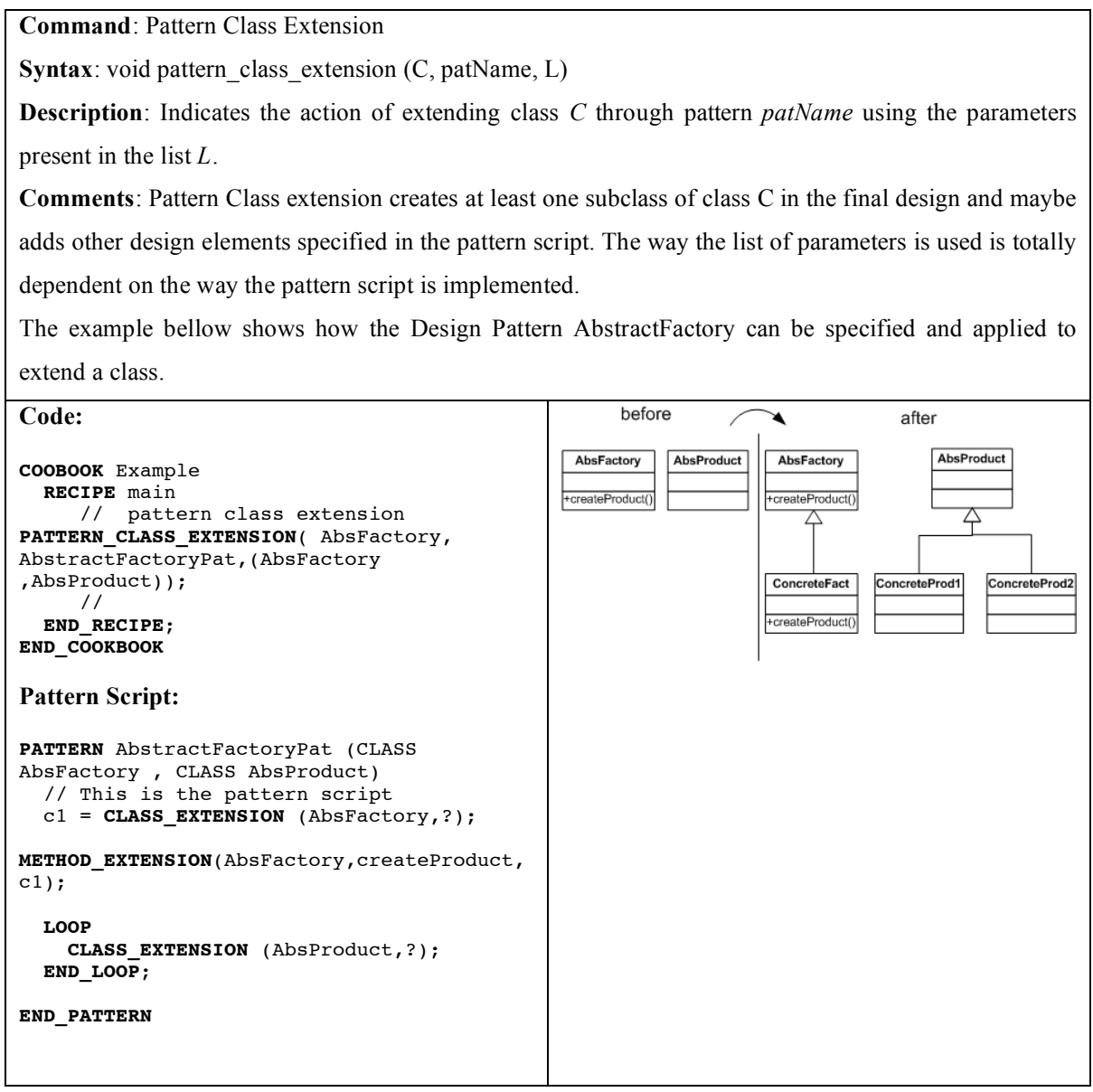

Table 14 - Pattern Class Extension Command 
Command: Pattern Method Extension

Syntax: void pattern_method_extension (superC, metName, subC, patName, list)

Description: Indicates the action of redefining method metName in subclass subC through pattern patName using the parameters present in the list $L$.

Comments: Pattern Method extension redefines the method metName in subclass subC and maybe adds other design elements specified in the pattern script. The way the list of parameters is used is totally dependent on the way the pattern script is implemented.

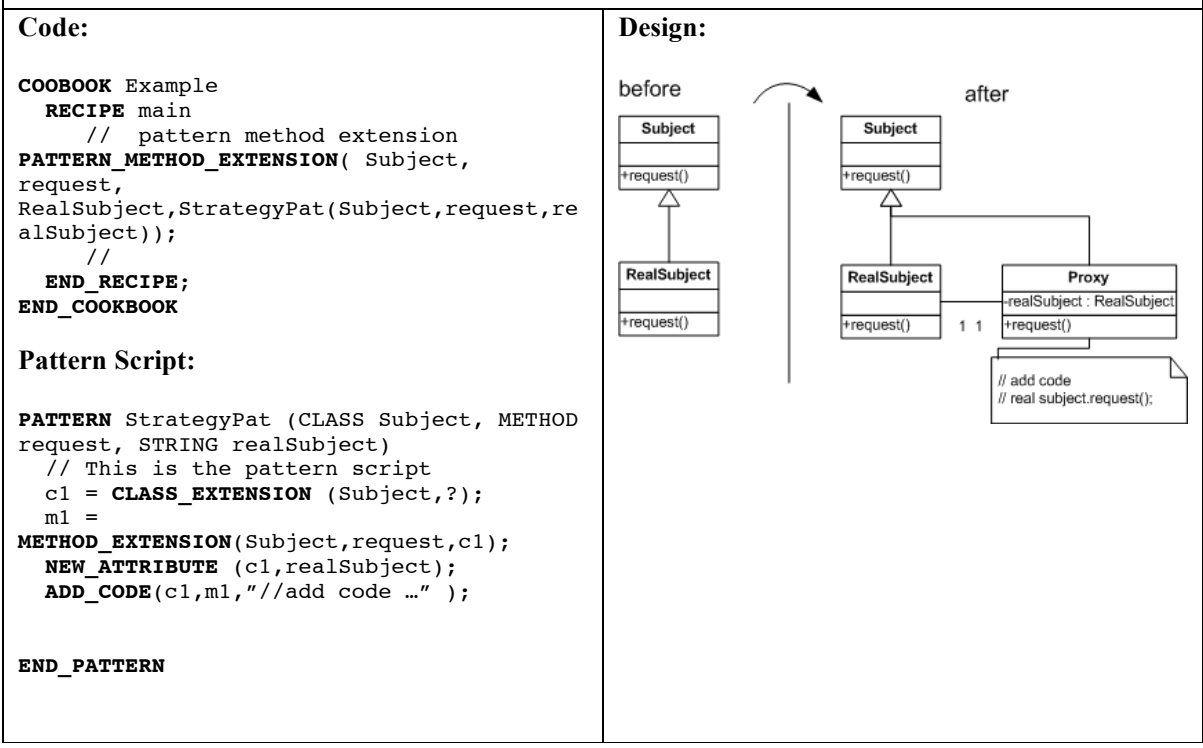

Table 15 - Pattern Method Extension Command

\subsection{Sequence Specific Tasks}

Sequence Specific Tasks (SSTs) describe the way RDL commands are combined in the execution flow (Tables 16 to 22). As in most imperative languages, commands need to be organized in a special order so that a particular computation occurs. For instance, when adding two numbers, someone needs to know their values prior to calling the sum operation. Instantiation processes are no different since instantiation tasks must tailor designs in an order that is stipulated by the framework developer.

Besides sequencing, which is related to order dependency, RDL also allows the specification of state dependency indicating if a design element is required in the final 
application. The main difference between the two dependencies is that state dependencies do not enforce a specific execution order between two or more commands, but only state that the commands must be executed, which is quite a common situation when instantiating frameworks used in product lines based on blackbox components.

It's worth mentioning that SSTs are represented using the infix notation for syntactic compliance to languages as $\mathrm{C}++$ and Java.

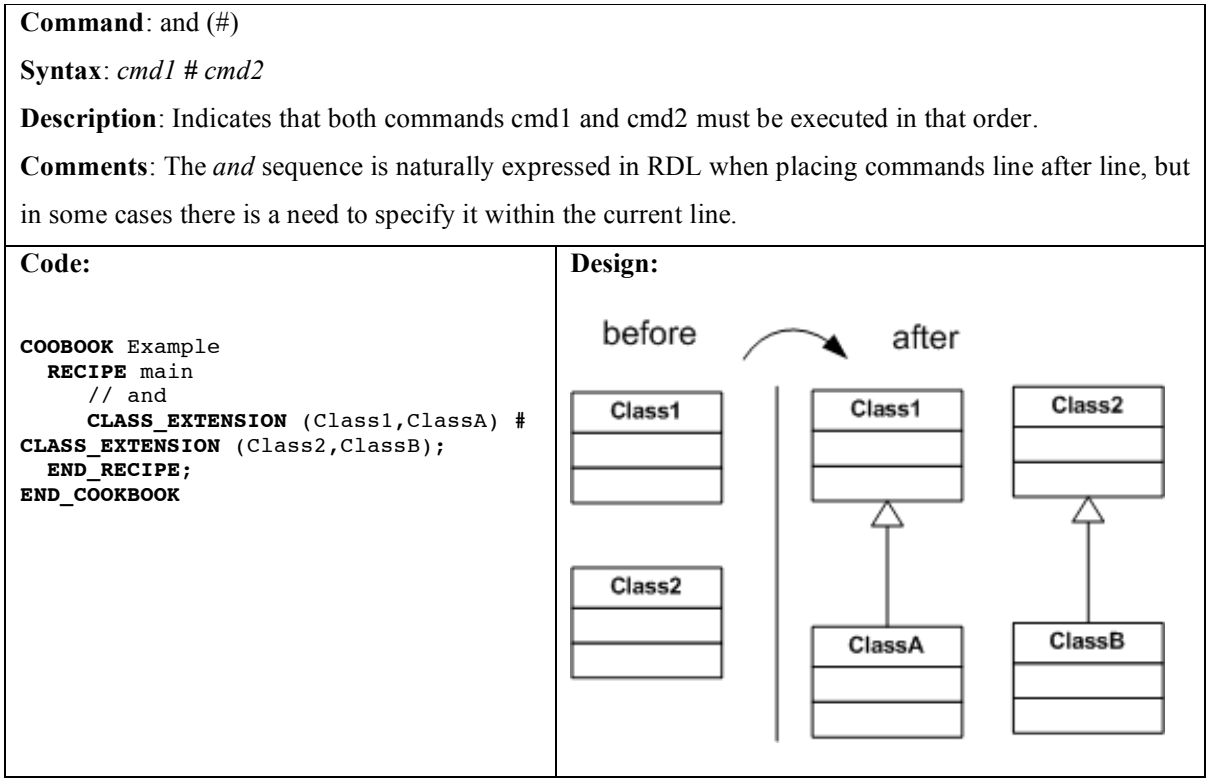

Table 16 - and Command

Command: or (o)

Syntax: $c m d 1$ o $c m d 2$

Description: Indicates that only one command should be executed.

Comments: The or sequence interrogates the framework re-user which option he/she is willing to execute. 


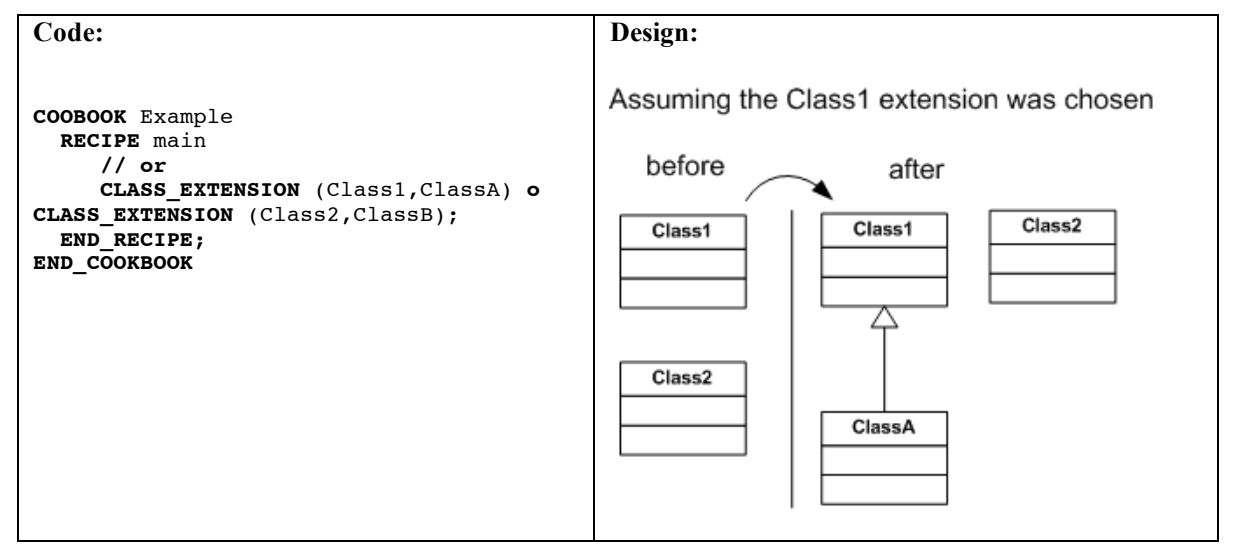

Table 17 - or Command

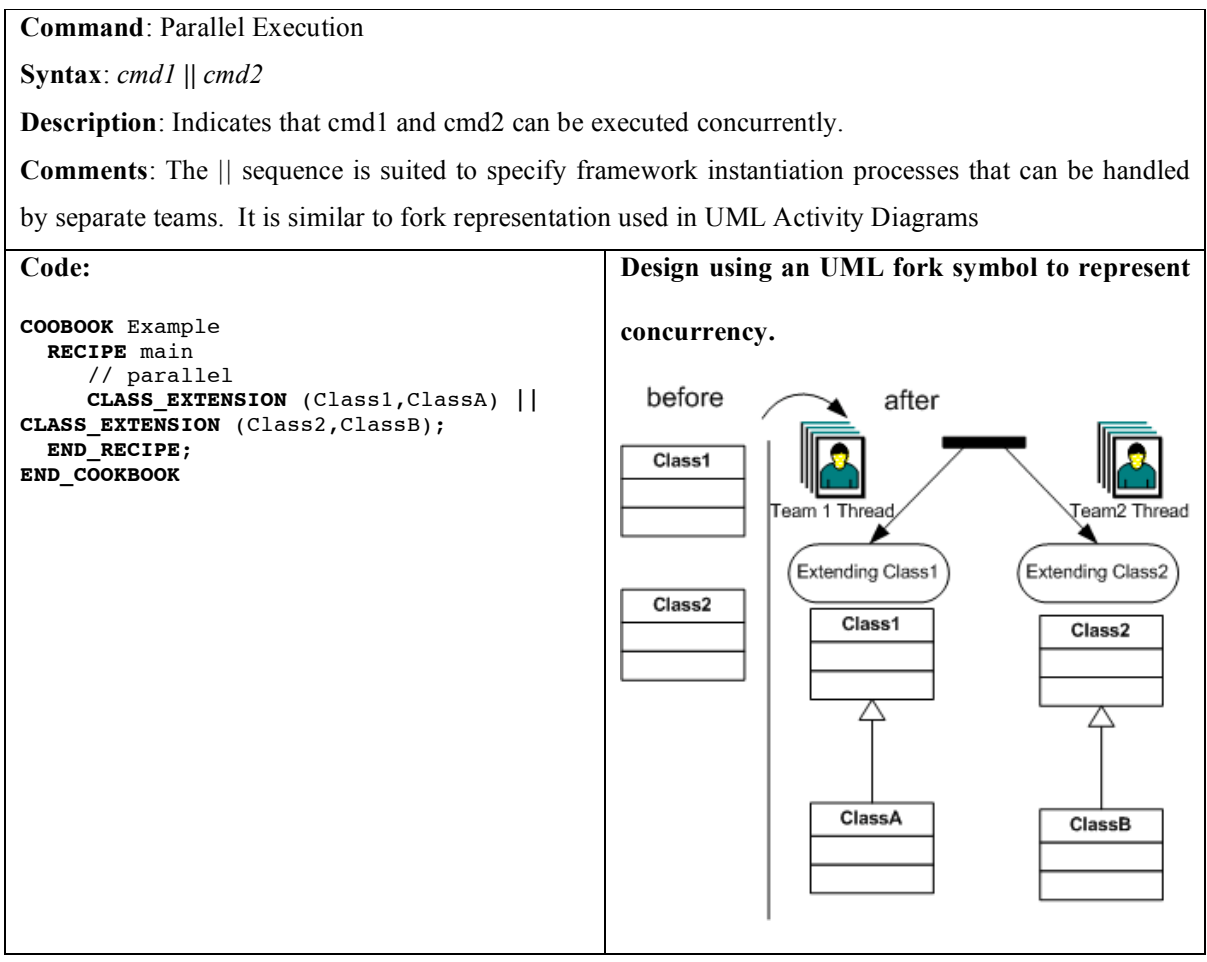

Table 18 - Parallel Execution Command 


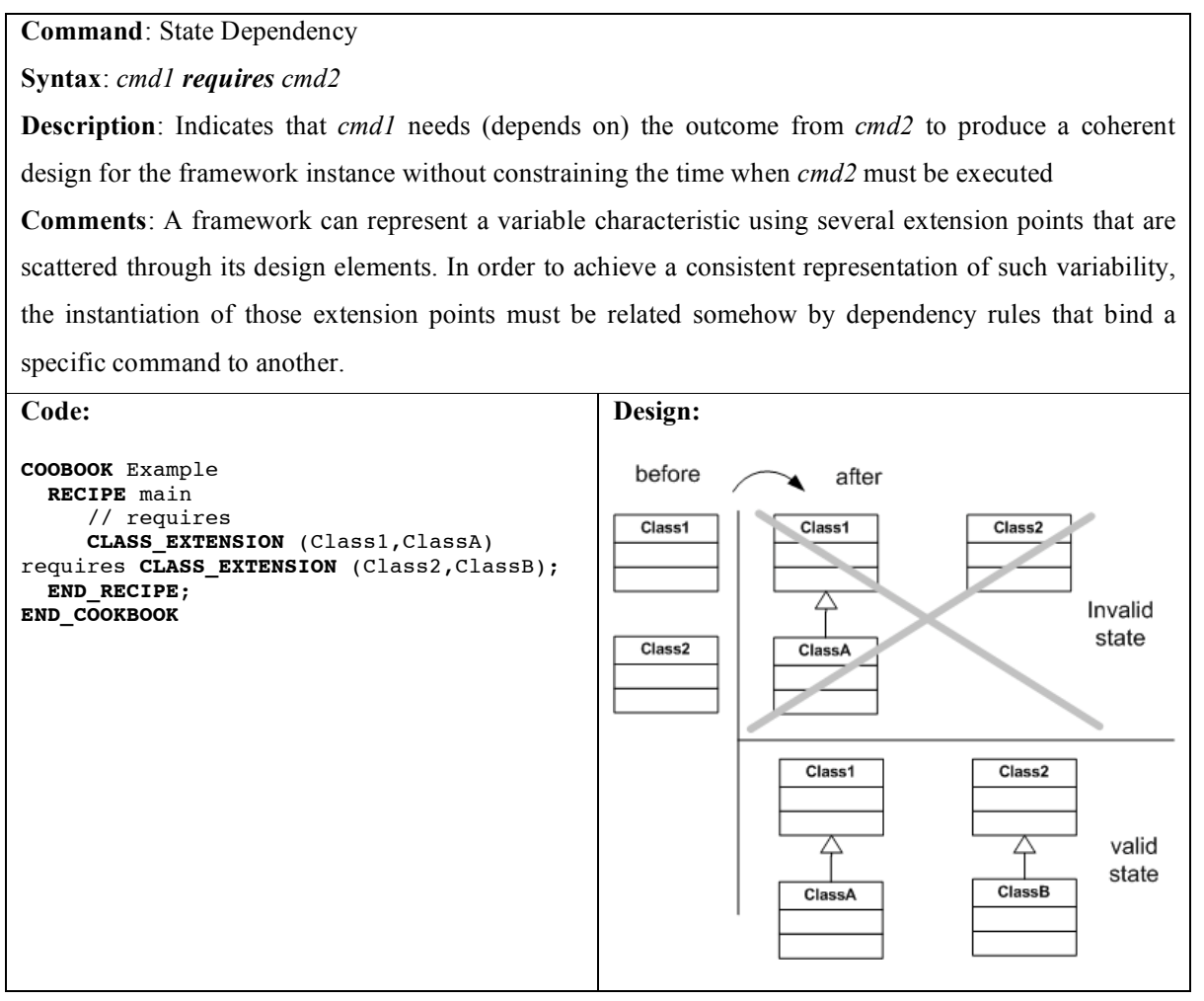

Table 19 - State Dependency Command

Command: Order Dependency
Syntax: $c m d 1$ requires before $\mid$ requires after $c m d 2$
Description: Indicates that a time dependency between $c m d 1$ and $c m d 2$. Requires before indicates that
$c m d 1$ must be executed previous to $c m d 2$. Requires after indicates $c m d 1$ must be executed subsequent to
$c m d 2$.
Comments: Order dependency commands have the same rationale of state dependency commands along
with a time constraint described in the commands execution flow.




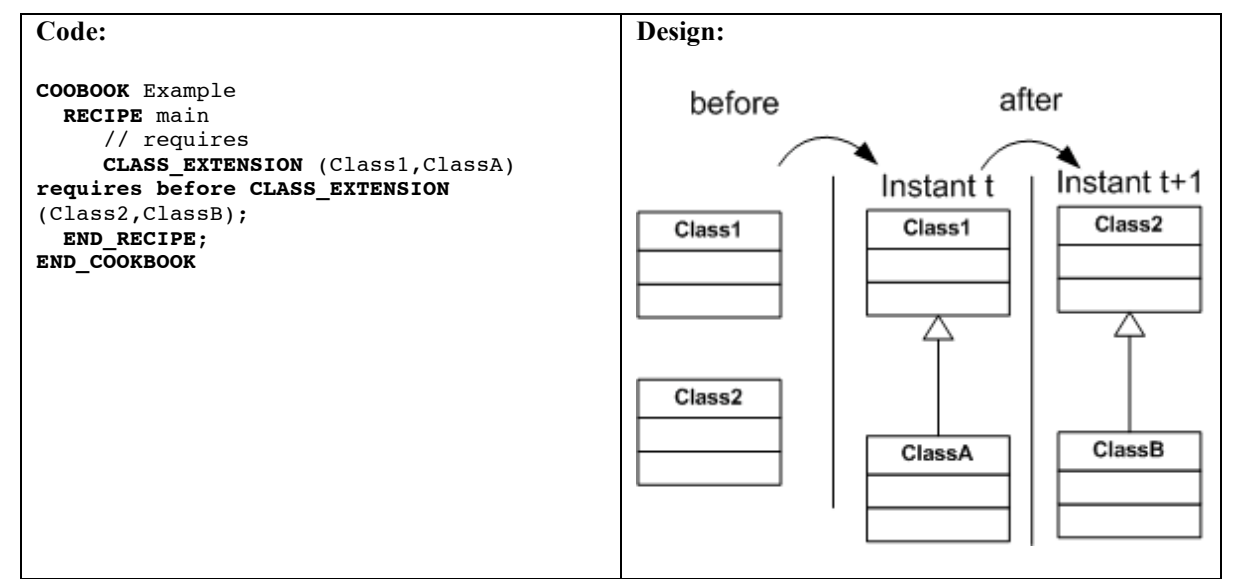

Table 20 - Order Dependency Command

Command: Synchronicity

Syntax: cmd 1 requires sync cmd2

Description: Indicates that the execution of $\mathrm{cmd} 1$ should be synchronous to $\mathrm{cmd} 2$, i.e., the instantiation process cannot continue till both commands are completed.

Comments: The requires sync command is suited for the synchronization of parallel instantiation processes that can be handled by separate teams. It is similar to join representation used in UML Activity Diagrams 


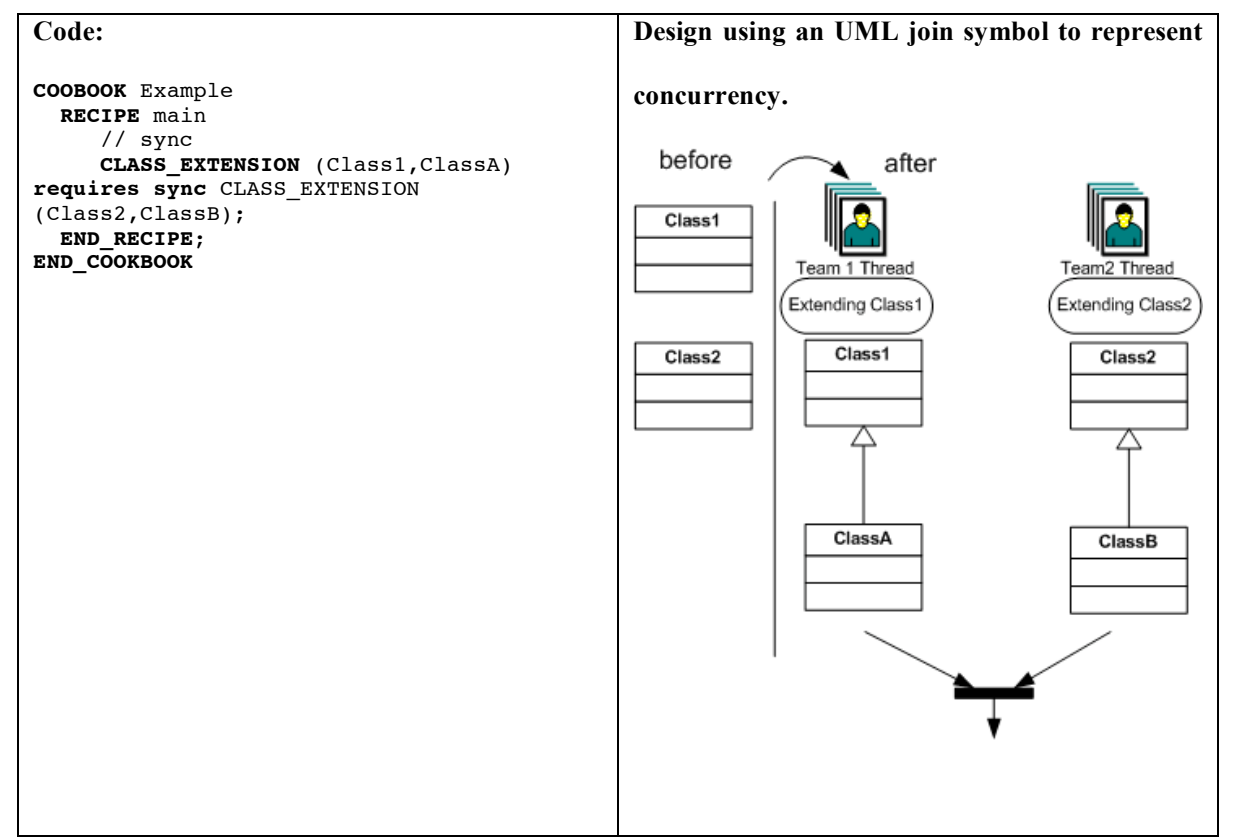

Table 21 - Synchronicity Command

Command: Mutual Exclusion

Syntax: cmd 1 requires exclusive $\mathrm{cmd} 2$

Description: Indicates that the execution of $c m d l$ and $c m d 2$ are mutually exclusive, i.e. if $c m d l$ is present in the final design, $c m d 2$ must not.

Comments: Extension points can represent alternatives in a domain that can not share the same solution space (framework instance) due to functional incompatibility. 


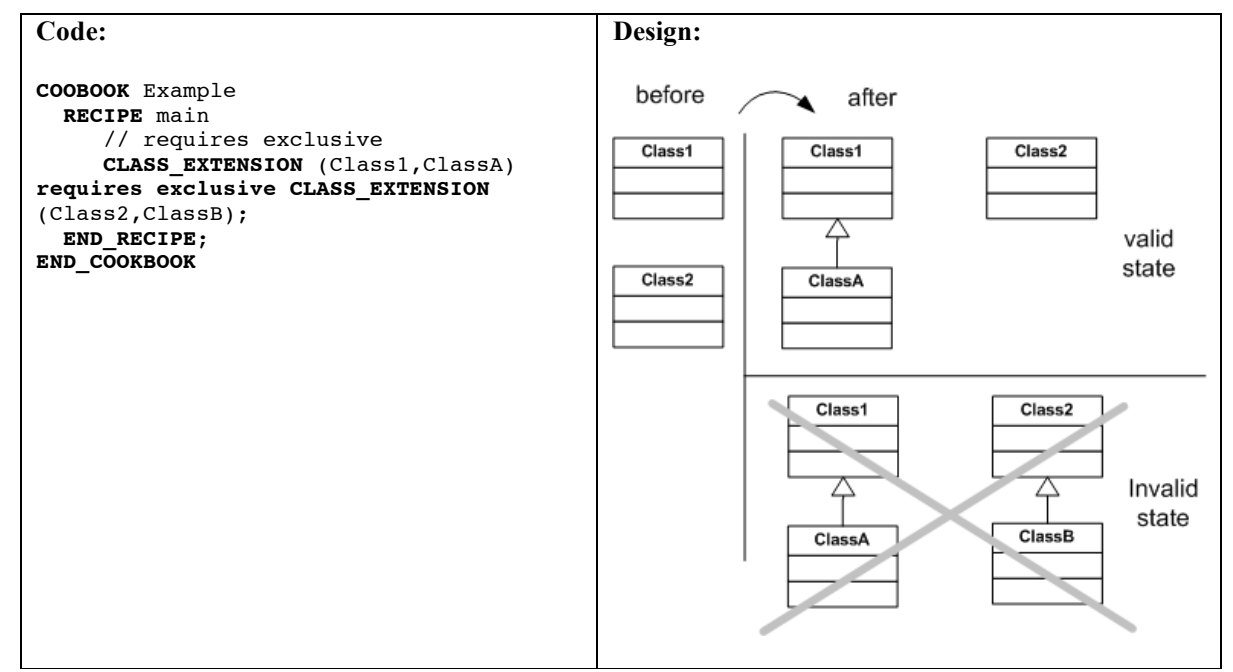

Table 22 - Mutual Exclusion Command

\subsection{Miscellaneous Tasks}

Miscellaneous Tasks (MT) are those tasks that do not fit well in the groups previously mentioned. They are typically related to RDL completeness as an imperative language

(Tables 23 to 25 ).

Command: Repetition

Syntax: loop cmds end_loop

Description: Indicates that $c m d s$ are repeated a number of times.

Comments: The number of iterations is defined by the re-user when interacting with the RDL execution environment. 


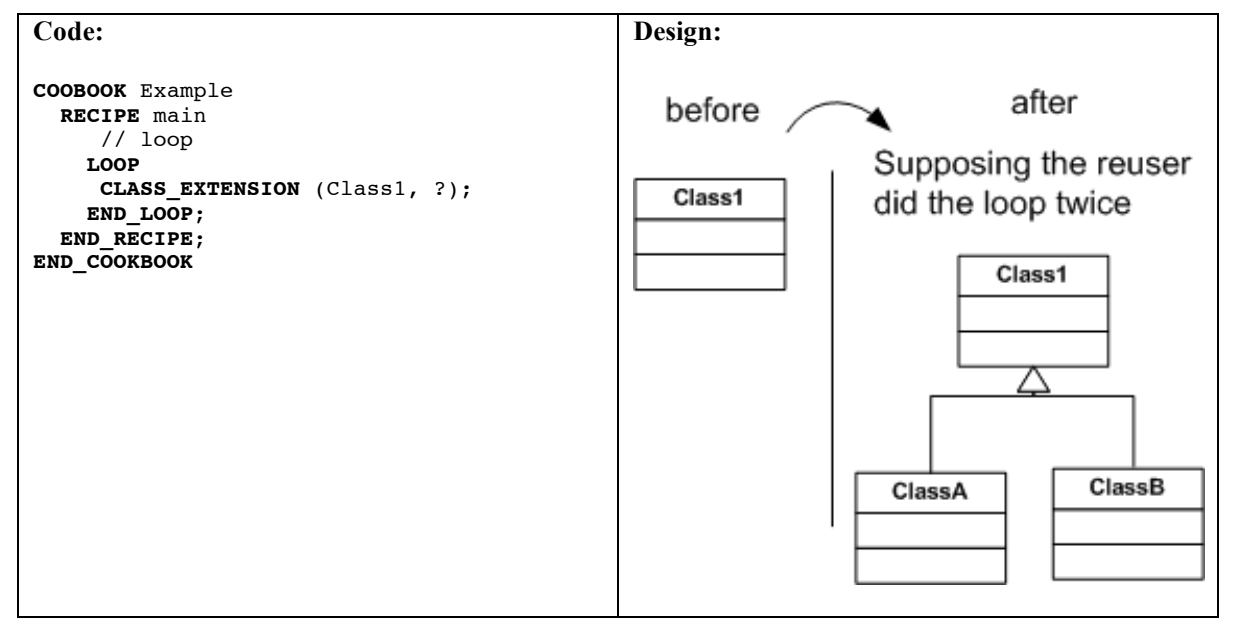

Table 23 - Repetition Command

Command: Assignment

Syntax: $v a r=c m d 1$

Description: Indicates that the result of execution of $c m d l$ is stored in var.

Comments: Some commands return the value of a particular execution. Storing such value for further use in the instantiation script helps in linking commands in an organized way.

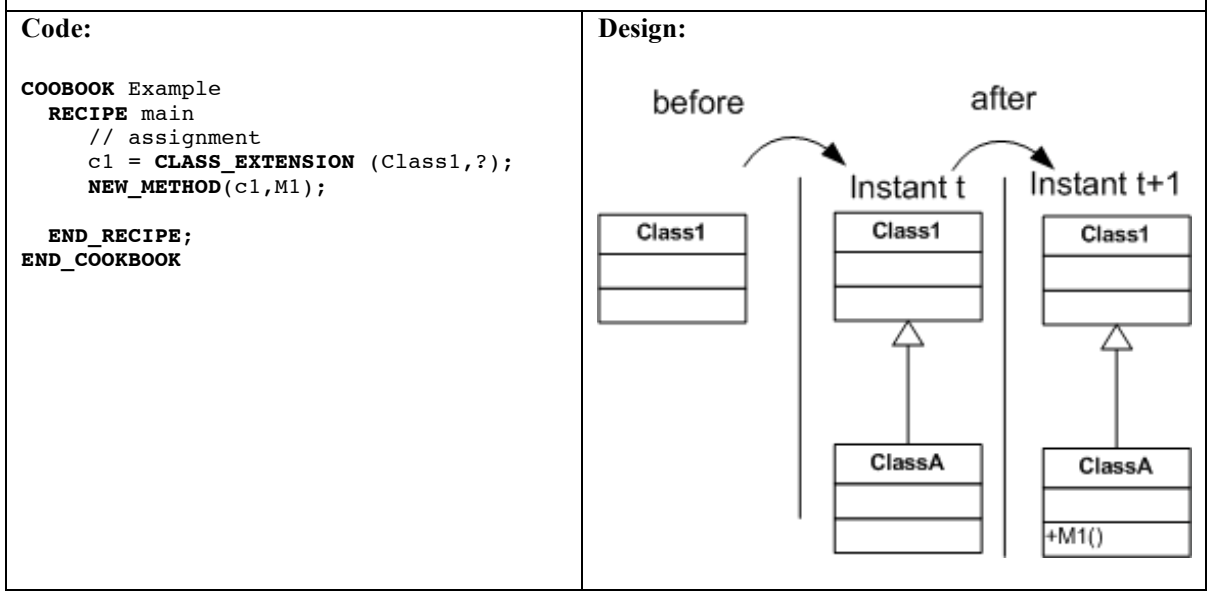

Table 24 - Assignment Command 


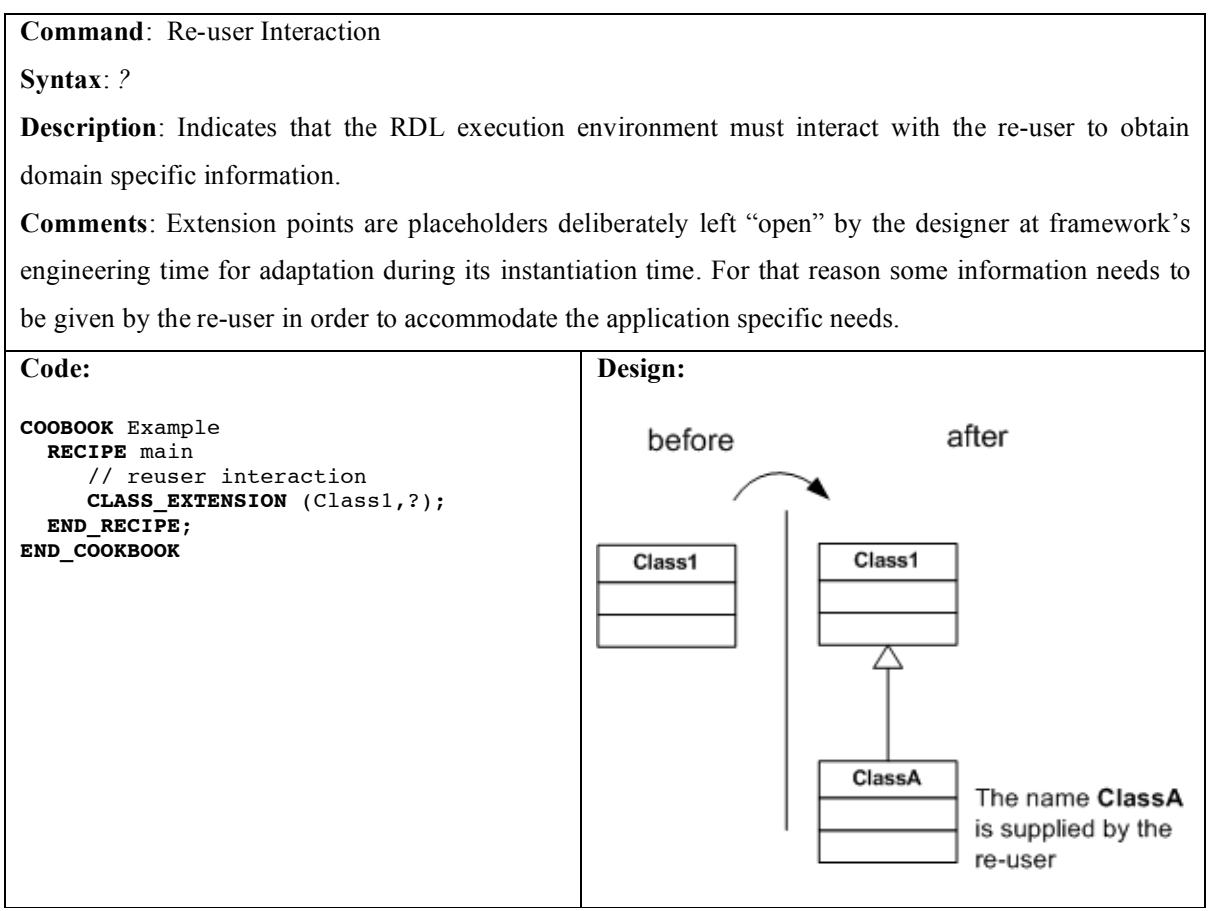

Table 25 - Re-user Interaction Command

\subsection{Recipes, Parameters \& Types}

RDL also allows the specification of procedures called Recipes. A recipe is used to modularize RDL scripts, thus improving readability. As shown in Table 26, a Recipe has a name, a list of parameters and a set of commands (i.e., recipe_body).

Command: Recipe

Syntax: $\boldsymbol{R E C I P E}$ name (parameter_list) recipe_body $\boldsymbol{E N D} \boldsymbol{R} \boldsymbol{E} \boldsymbol{C I P E}$;

Description: Specifies a recipe im RDL.

Comments: Allows modularization of RDL scripts. 


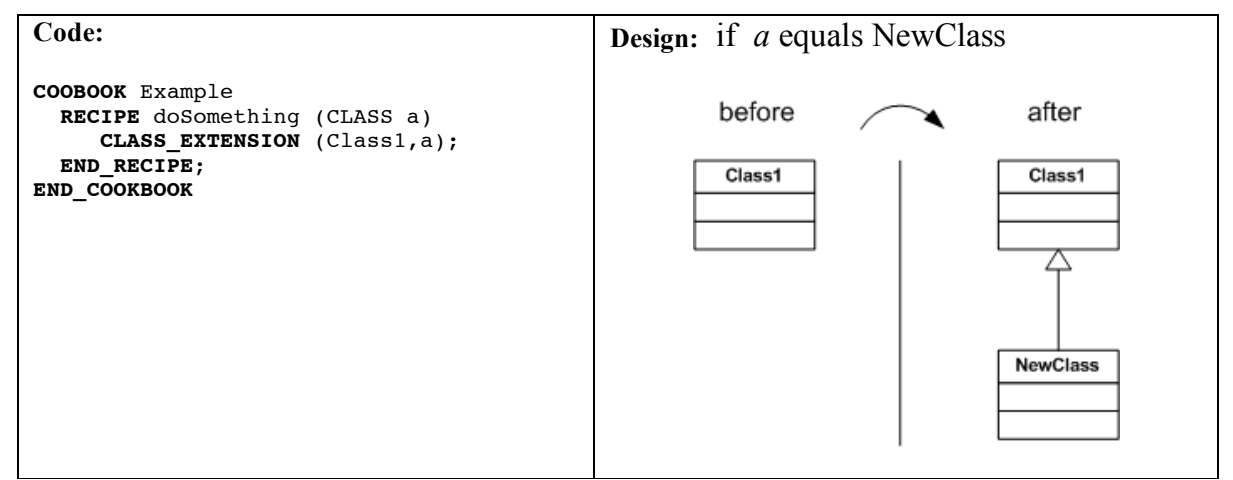

Table 26 - Recipe Example.

In order to invoke a recipe an RDL programmer should only state its name and required parameters (see Table 27).

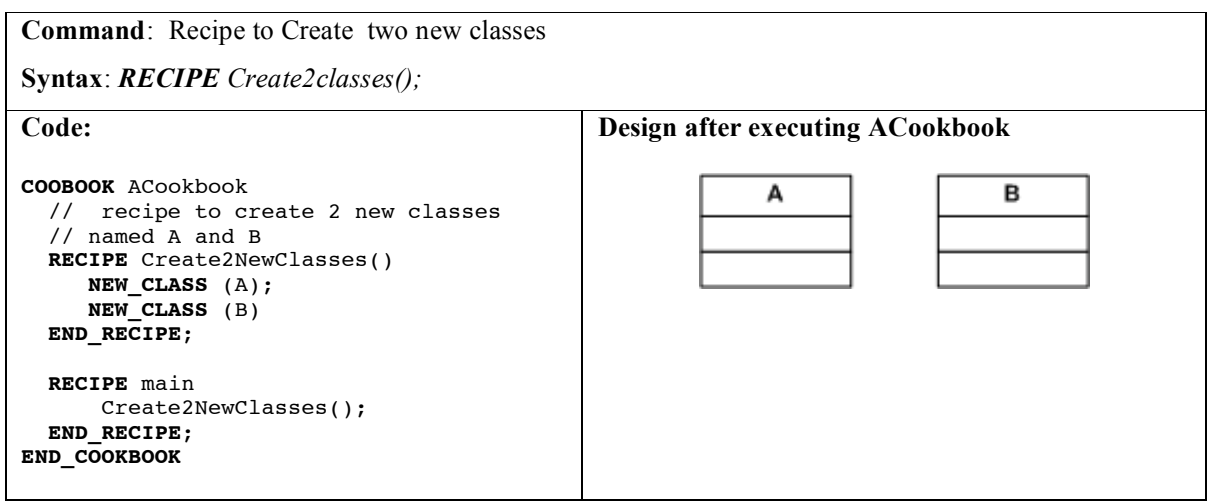

Table 27 Recipe Invocation.

In the current version of RDL we did not adopt strong typing since the result variables declared as part of an assignment command and parameters used in Recipes do not need to be typed. Not typing variables and parameters simplified the implementation of the RDL execution environment.

On top of the Recipes foundation we have created the concept of Pattern scripts. Patterns are used as placeholders for framework instantiation activities that solve recurring instantiation problems. For example, when instantiating HotDraw [9], for every drawing 
element that a HotDraw instance makes available on its palette, it is mandatory to provide its name, its icon and its associated drawing tool. Thus, representing such activities using the RDL rationale leads to creating a Pattern script (Table 28) that gathers the commands required to adapt the HotDraw model. It's worth mentioning that Patterns are supposed to represent problem-solution structures to facilitate the match between application needs and the framework's concepts. For instance, in the case of Hotdraw, which is a framework for drawing, the most common problem is "How do I create new Figures?" and so a pattern can help solve this problem.

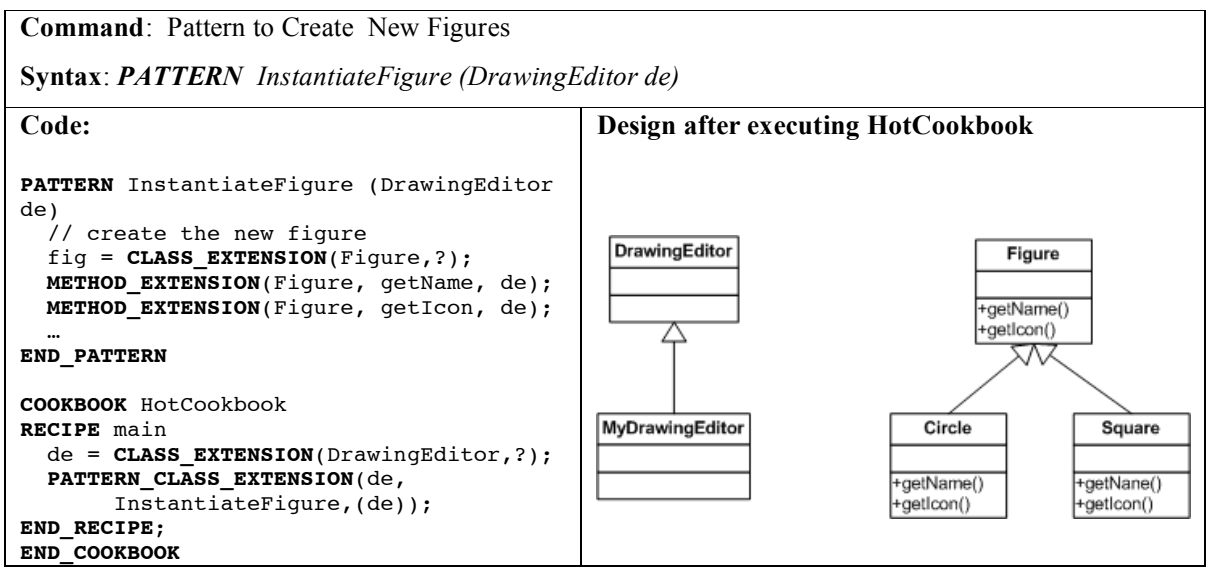

Table 28 - Pattern Example.

\section{RDL Execution Model}

The RDL approach is intended to assist the framework instantiation process by providing a script and underlying software system to run and control the instantiation activities. For this reason, RDL needs an underlying execution environment capable of transforming commands into a set of low level instructions that change the state of inputs, which in our case are UML models. 
In order to organize the RDL execution environment we have developed a Java-based prototype specified in the architecture shown in Figure 6: a Compiler, a Virtual Machine, the XMIProxy, a UserInterface and RuntimeLibrary.

The RDL Compiler validates RDL scripts and prepares a set of information required for execution. It starts by analyzing the script's lexical and semantic structure to check if the language tokens and commands are properly used. Then, this compiler creates a table with instantiation instructions by compiling high-level instantiation commands, such as a CLASS_EXTENSION, into a sequence of low-level commands, such as a NEW_CLASS, NEW_INHERITANCE pair for this case. In addition the compiler generates additional information such as the variables allocation list and the state and order dependency list.

Once the input stream is validated and the low-level information is in place, the RDL Virtual Machine (RVM) can start by executing the set of instructions found in the Instantiation Instruction table as shown in Figure 6. During execution, the RVM must tailor the UML model and incorporate the new design elements. This step can be achieved by manipulating the UML XMI representation as an XML document using translators written in XSL [40]. However, as we find the manipulation of XMI in its "natural form" cumbersome and, given that the relationships among design elements are scattered in the representation and the elements are indirectly accessed via several IDREF tags, we have developed the XMIProxy component, which is responsible for creating an in memory version of an UML model represented in XMI. 
The XMIProxy component houses the code required for XMI manipulation. It is capable of reading/writing XMI files and creating an in-memory class space, containing the classes, methods and attributes for the input model. By adding new elements to the class space by invoking the XMIClass.createClass() method, it is possible to manipulate the model using the RDL script commands.

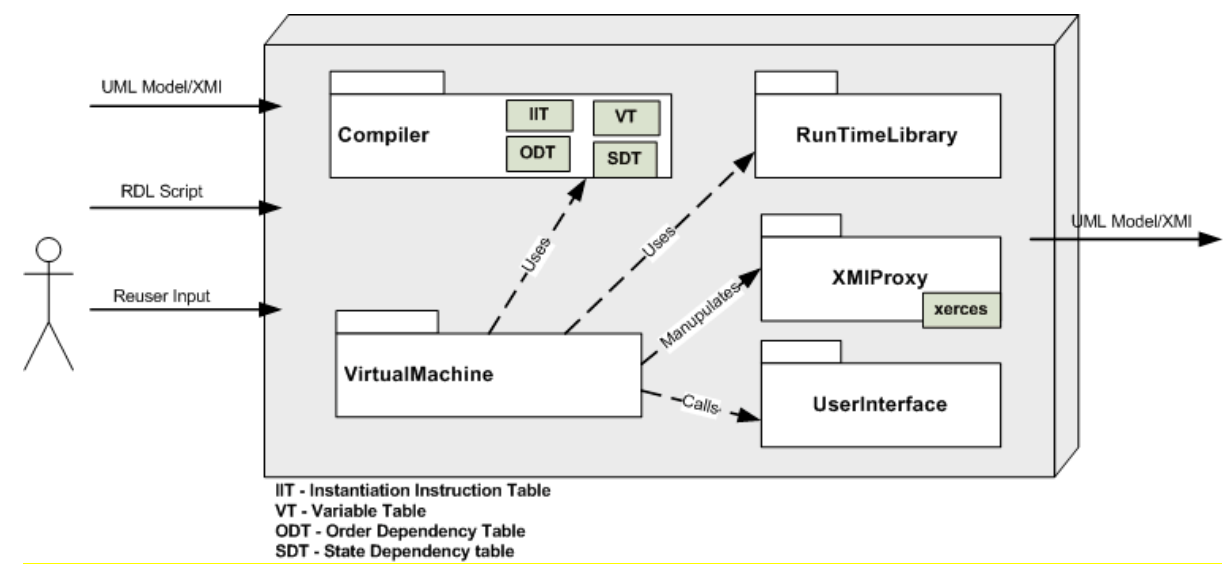

Figure 6 - RDL Execution Environment.

The RDL environment also has a UserInterface library where the dialogs required for reuser input (e.g., prompting the re-user for a new class name) are defined.

To run this environment a re-user must specify two input files ${ }^{5}$, one with the RDL script and the other with the framework design expressed in UML-FI, which is XMI. The XMI file will then be manipulated and saved as a new file preserving the original. Since the

\footnotetext{
${ }^{5}$ Those are the files used by the RDL execution environment and that is why the Features Model is not used here.
} 
process is not totally automated, during its execution the re-user also needs to provide some information to the RDL environment, indicating items such as new names, when to finish a loop, or which path to follow in a OR command.

\section{The JUnit Illustrative Example}

This section provides several instantiation scripts to show how RDL can be used to tailor framework design. We have chosen JUnit Version 3.8 [37] for this illustration, as it has substantial documentation and tool support. Although JUnit is a small framework with a few design elements (approximately 9 classes and 50 methods), it is widely used by the software developer community and thus it is used to demonstrate the rationale behind various instantiation process scripts.

\subsection{JUnit Specification}

JUnit is an object-oriented whitebox framework [1] built to provide developers with a standard and easily used interface for defining (coding) and running test cases. JUnit's functionality is expressed in a relatively simple design as presented in Figure 7. 


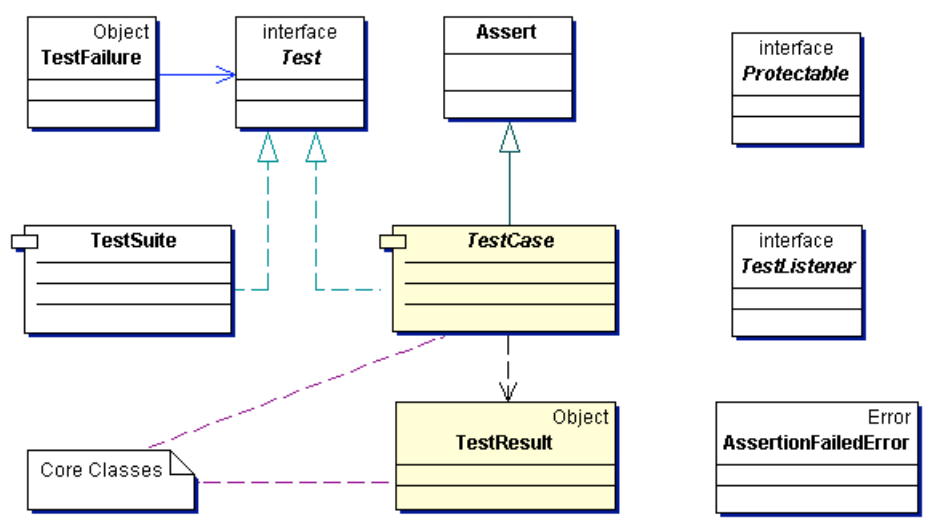

Figure 7 Junit design.

Taking into account JUnit's reuse specification (instantiation process), its design can be reduced to two classes: TestCase and TestResult (Figure 8).

$\Rightarrow$ TestCase - Provides placeholders (methods to be extended) for test coding and running. Its reuse is mandatory and must be done through inheritance and method redefinition.

$\Rightarrow$ TestResult - Collects test results and presents them in a textual fashion. Its reuse is optional and must be done through inheritance and method redefinition. 


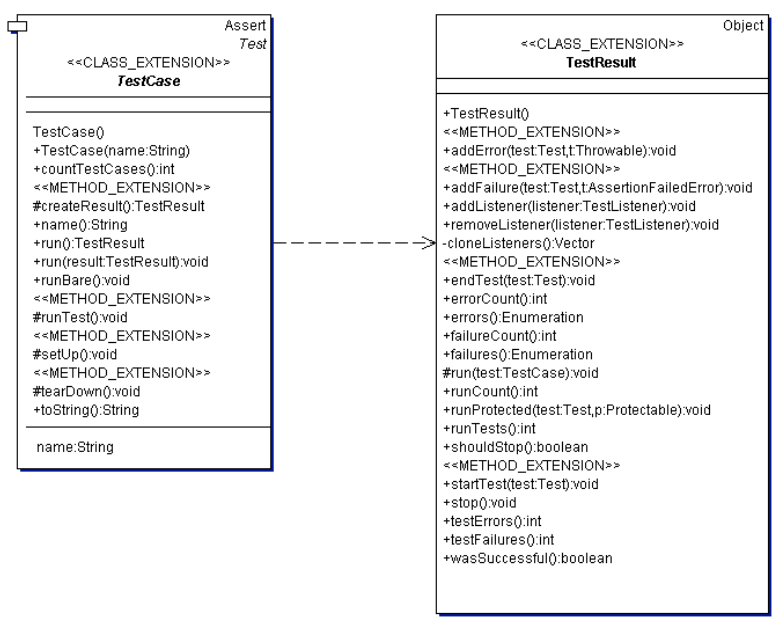

Figure 8 - UML-FI Diagram specifying JUnit's Extension Points.

Although JUnit's reuse deals with few classes, the re-user may have to manage different reuse strategies and different reuse restrictions. According to its documentation, JUnit can be applied in a simple test or in a fixture for multiple tests; it can change the appearance of the Result or be applied in complex development environments. Each of these reuse strategies has its pitfalls and should be specified carefully to provide an accurate JUnit instance.

In the next sections we provide a thorough representation of each JUnit reuse strategy accompanied by the corresponding UML-FI representation and RDL script.

\subsection{TestCase - One Single Test}

\subsubsection{Instantiation Specification}

The specification was extracted from the junit.org cookbook [37]: 
$\Rightarrow$ Create an instance of TestCase () - In RDL means subclass TestCase()

$\Rightarrow$ Override the method runTest() and fill it with test code

\subsubsection{UML-FI Representation}

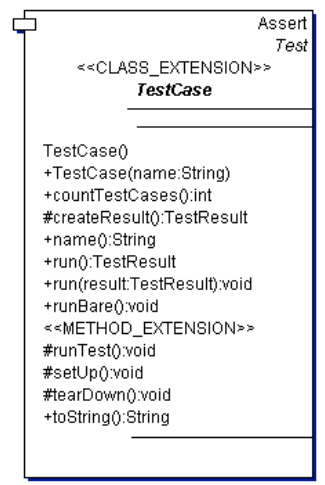

\subsubsection{RDL Script}

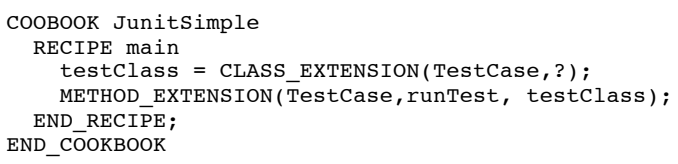

Code 2 RDL Script to instantiate JUnit

\subsection{Fixture-Multiple Tests}

\subsubsection{Instantiation Specification}

The specification was extracted from the junit.org cookbook [37]:

$\Rightarrow$ Create a subclass of TestCase

$\Rightarrow$ Override setUp() to initialize the variables

$\Rightarrow$ Override tearDown() to release any permanent resources you allocated in setUp

$\Rightarrow$ Add an instance variable for each part of the fixture

$\Rightarrow$ For each test implement a method which interacts with the fixture 


\subsubsection{UML-FI Representation}

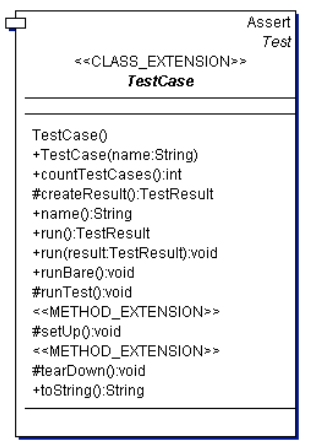

\subsubsection{RDL Script}

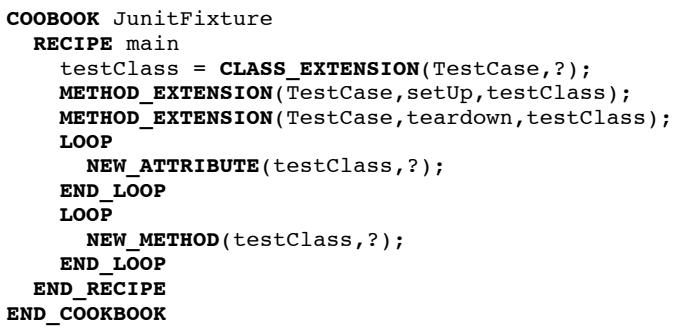

Code 3 RDL Script for Fixture Example

\subsection{TestResult - Changing Results}

\subsubsection{Instantiation Specification}

The specification was extracted from the junit.org cookbook [37]:

$\Rightarrow$ Create your Testcase class as above

$\Rightarrow$ Create a subclass of TestResult

$\Rightarrow$ Add a new method to meet your requirements

$\Rightarrow$ Override createResult

$\Rightarrow$ Instantiate the new TestResult subclass with the overridden createResult 


\subsubsection{UML-FI Representation}

\begin{tabular}{|c|c|}
\hline $\begin{array}{r}\text { Assert } \\
\text { Test } \\
\Leftrightarrow \ll \text { CLASS_EXTENSION }>>\end{array}$ & $\begin{array}{c}\Leftrightarrow \ll \text { CLASS_EXTENSION } \Rightarrow> \\
\text { TestResult }\end{array}$ \\
\hline 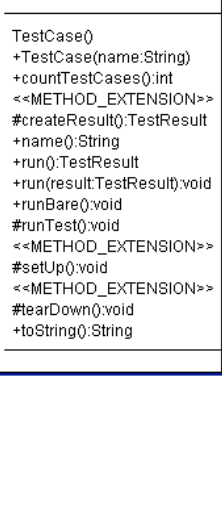 & 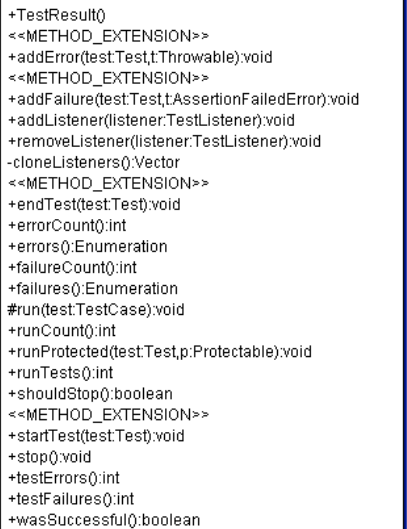 \\
\hline
\end{tabular}

\subsubsection{RDL Script}

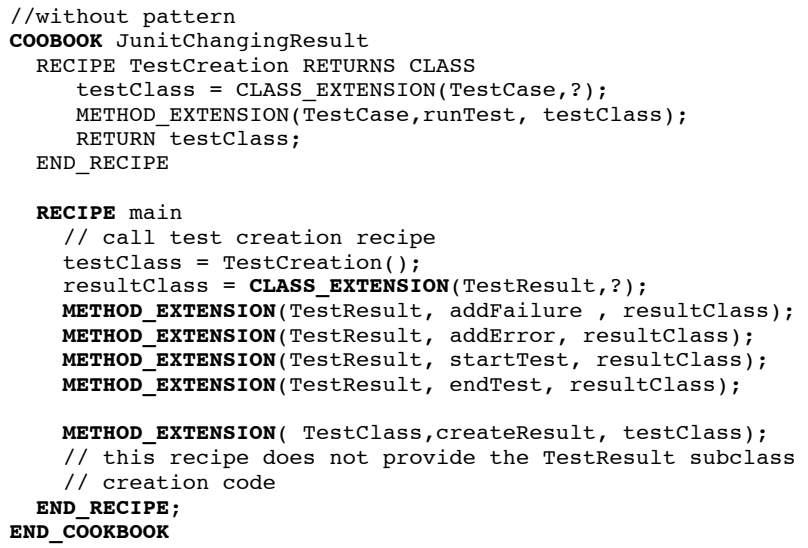

\section{Code 4 RDL Script for the Changing Result example}

We can modify the JunitChangingResult cookbook to illustrate the use of a pattern instantiation that will reduce the effort needed to obtain the final application. According 
to JUnit's documentation, the sub-classes from TestClass and TestResult must be connected at the code level during the execution of the method createResult, It is not possible to make this connection at Framework Engineering Time because the subclasses' names are not known. This problem was solved by adopting the Design Pattern Factory [41] that was created to defer instantiation to subclasses.

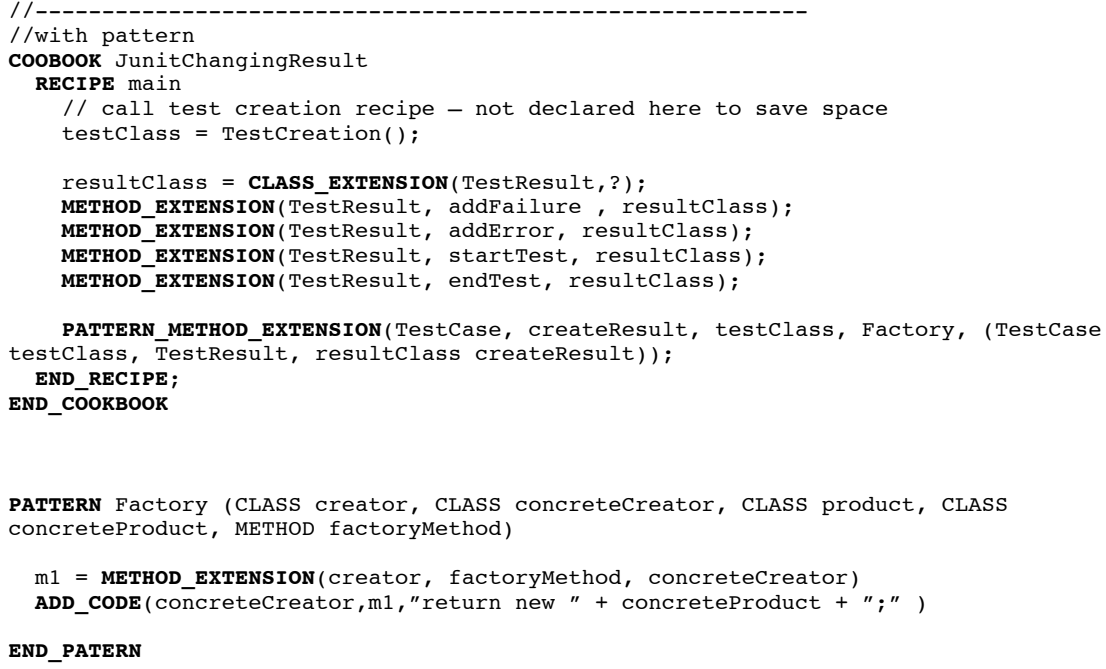

Code 5 RDL Script for the Changing Result Example using the Factory Pattern.

\section{Conclusions and Future Work}

In this paper we present RDL (Reuse Description Language), which is an approach to specify framework instantiation processes. RDL is an imperative language that supports representing framework instantiation activities in a script form named Cookbook that can be processed by a tool, thus guiding re-users through the instantiation process. Instantiation activities in RDL are related to design manipulation and sequencing, 
tailoring designs at specific extension points by means of typical object-oriented programming activities.

An important aspect of our approach is the ability to represent concurrent instantiation activities, which facilitates the specification of distributed instantiation processes that are widely used in the Global Software Development scenario for reducing development costs [3].

Compared to approaches such as Cookbooks[9] and Hooks [10], RDL provides an explicit and a more precise way to represent instantiation activities since it does not use natural language in its representation but uses a Domain Specific Language carefully developed for the instantiation domain. As a result, RDL scripts can be processed by tools, facilitating the development of Instantiation Wizards. Moreover, because of the nature of RDL and the extensive use of XML technologies, such wizards do not need to be hard-coded within a specific tool as is common in Product Lines approaches such as WebML[18] and Pulse[38]. Instead RDL-based wizards can be developed declaratively as components in Product Line Architecture environments.

There are many differences between RDL and model transformation approaches such as UMT-QVT[42], Velocity[43], XSLT and MTL[44]. UMT-QVT works with a compact version of XMI, the XMI-Lite, and creates an environment to encapsulate transformations that can be specified as Java programs or XSTL scripts. However, UMTQVT is not suitable for handling re-user interactions and state/order dependency. XSLT 
has a broader use since it can be adopted to manipulate any XML file, although it suffers from the same issues related to UMT.

Velocity [43] is a Java-based template engine supporting the definition of templates with the VTL (Velocity Template Language). Using VTL to transform XMI files is cumbersome since the commands must be embedded into a file usually with several lines in XML. Moreover, VTL is designed as a one-pass transformer and thus leaves no space for re-user interaction.

MTL [44] encloses some of our ideas although it was created as a full transformation language. For that reason MTL is a broader language in terms of concepts and has no special functionality to framework instantiation.

Regarding RDL usage, we have conducted experiments with frameworks other than JUnit to validate its applicability and efficacy. In [39] the author reported the use of RDL to specify the instantiation process for the Web-based search engine Avestruz [24] and for the VMarket eCommerce [25] frameworks. In [33] the author presented the use of RDL to instantiate the drawing tool framework. One of the most important results of RDL usage can be found at [22] where the author indicates that the use of instantiation patterns Toacy C Oliveira 8/1/06 5:29 PM Deleted: 44

brought an increase in understanding the framework's domain characteristics and also avoided instantiation inconsistencies since the hotspots are pinpointed by the RDL script. Note, however, that since the RDL tool is not fully functional, in all these cases the XMI file generated by the RDL environment needed some minor modification. For instance, 
we did not handle the code assignment command properly as indicated in the UML metamodel and, therefore, a return parameter must be completed by the re-user.

In future work, we want to incorporate the concept of strong typing into RDL. In this version of RDL, variables have their types dynamically assigned depending on the use. For instance, a variable $c 1$ can be used to hold the return of a CLASS_EXTENSION command, which returns a class, and in the next line of the same script the same $c 1$ variable can be used to hold the return of a METHOD_EXTENSION command, which returns a method. When instantiation scripts grow larger, it becomes hard to "discover" the type of a variable by inspecting the whole script and for that reason we plan to modify RDL to accommodate variable declaration. Some preliminary work in this direction is presented in [22]. We are also extending our approach to handle cross-cutting concernbased extensions and define aspect-oriented framework instantiation [46].

\section{Acknowledgements}

This research was supported by the Natural Sciences and Engineering Research Council of Canada (NSERC) and the National Brazilian Research Council (CNPq).

\section{References}

1. Fayad, M.F., Schmidt, D.C., Johnson, R.E., Building Application Frameworks: Object-Oriented Foundations of Framework Design, John Wiley and Sons, 1999.

2. Tomer,A., Goldin,L., Kuflik,T., Kimchi E., Schach S.R., Evaluating Software Reuse Alternatives: A Model and Its Application to an Industrial Case Study IEEE Transactions on Software Engineering, September 2004 vol. 30 n.9 p601-612

3. Herbsleb J.D., Mokckus, A., Finholt T.A., Grinter R.E., An Empirical Study of Global Software Development: Distance and Speed, p81-90, International Conference on Software Engineering, Toronto, Canada May 2001. 
4. Batory, D., Cardone, R., Smaragdakis, Y.,Object-Oriented Frameworks and Product Lines,Proceedings of the First Software Product Line Conference,p227--247, 2000

5. Oliveira, T.C., Alencar, P., Cowan, D. Filho, I.M., Lucena, C.J.P. Feature Driven Framework Instantiation In: Ecoop, 2003, Darmstad. Workshop on Modeling Variability for Object-Oriented Product Lines. , 2003.

6. Oliveira, T.C., Alencar, P., Cowan, D., Filho, I.M., Lucena, C.J.P., Software Process Representation and Analysis of Framework Instantiation, IEEE-Transactions in Software Engineering, March 2004 p145-159

7. Filho, I. F., Oliveira, T. C., Lucena, C. J. P. A Framework Instantiation Approach Based on the Features Model. Journal Of Systems And Software v. 73, n. 2, p. 333-349, September 2004.

8. Oliveira, T.C., Alencar, P., Cowan, D. Filho, I.M., Lucena, C.J.P Enabling Model Driven Product Line Architectures European Workshop on MDA Canterbury UK 2004

9. Johnson, R., Documenting Frameworks Using Patterns, Proceedings of OOPSLA'92, ACM/SIGPLAN, New York, 1992.

10. Froehlich, G., Hoover, H.J., Liu L. and Sorenson, P.G. Hooking into Object-Oriented Application Frameworks, Proc. 19th Int'l Conf. on Software Engineering, Boston, May 1997, 491-501.

11. Ortigosa, A., Campo, M., Smartbooks: A Step Beyond Active-Cookbooks to Aid in Framework Instantiation, Technology of Object-Oriented Languages and Systems 25, IEEE Press, June 1999.

12. V. Cechticky, E. Chevalley, A. Pasetti, W. Schaufelberger, A Generative Approach to Framework Instantiation, Erfurt, Germany, Proceedings of GPCE, pp. 267-286, LNCS 2830 OOPSLA

13. Oliveira, T.C., Alencar, P., Cowan, D. : Towards a declarative approach to framework instantiation Proceedings of the 1st Workshop on Declarative Meta-Programming (DMP-2002), September 2002,Edinburgh, Scotland, p 5-9

14. Gomaa, H. ,Designing Software Product Lines with UML, Addison Wesley Object Technology Series, July 2004

15. Czarnecki, K.. Overview of Generative Software Development. In Proceedings of Unconventional Programming Paradigms (UPP) 2004, 15-17 September, Mont Saint-Michel, France, Revised Papers, volume 3566 of Lecture Notes in Computer Science, pages 313-328. Springer-Verlag, 2004.

16. WebMl Documentation at www.webml.org accessed in November 2005

17. MDA Guide at http://www.omg.org/cgi-bin/doc?omg/03-06-01 accessed in November 2005

18. OCL Specification at http://www.omg.org/docs/ptc/03-10-14.pdf accessed in November 2005

19. QVT Request for proposal at ftp://ftp.omg.org/pub/docs/ad/02-04-10.pdf accessed in November 2005

20. Haeberer, A Maibaum T. The Very Idea of Software Development Environments: A Conceptual Architecture for the ARTS Environment Paradigm. ASE 1998: 260-271

21. Carvalho S.E.R.; Cruz, S.O.; Oliveira, T.C. Second Generation Object-Oriented Development US - Brazil Joint Workshops on the Formal Foundations of Software Systems Rio de Janeiro, May 5 9, 1997 and New Orleans, November 13 - 16, 1997

22. Mendonca, M., Oliveira, T.C., Alencar, P., Cowan, D., Assisting Framework Instantiation: Enhancements to Process-Language-based Approaches. Technical Report CS-2005-25 at University of Waterloo, School of Computer Science.

23. A. Garcia, M. Cortés, C. Lucena. "A Web Environment for the Development and Maintenance of E-Commerce Portals Based on Groupware Approach". Proc. of the IRMA'2001, Canada, May 2001.

24. Magalhães,J. Lucena,C.J.P. Using an Agent-Based Framework and Separation of Concerns for the Generation of Document Classification Tools, Lecture Notes in Computer Science, Volume 2926, Feb 2003, Pages 193 - 200

25. Lucena C.J. P. Ripper, P. Fontoura, M. V-Market: A Framework for Agent eCommerce Systems at www.almaden.ibm.com/cs/ people/fontoura/papers/www2000.pdf accessed in Novembre 2005

26. Oliveira, T.C. ; Carvalho, S.E.R. ;Lucena C.J. P. DSSFrame - A Decision Suppot System with Agents. Techinical Report 2000-18 Pontifícia Universidade Católica do Rio de Janeiro - Brazil 2000. 
27. Oliveira, T.C., Alencar, P., Cowan, D. Filho, I.M., Lucena, C.J.P. xTAO: enabling a declarative approach to the specification of multi-agent systems Proceedings of the fourth international workshop on Software engineering for large-scale multi-agent 2005 p1-7.

28. Mattsson, M. Evolution and Composition of Object-Oriented Frameworks, PhD Thesis, Department of Software Engineering and Computer Science, University of Karlskrona/Ronneby, 2000 .

29. Cheng, L.T., Souza, C.R.B., Hupfer, S. , Patterson, P. , Ross, S. , Building Collaboration into IDEs at http://www.acmqueue.com/modules.php?name $=$ Content\&pa $=$ showpage \&pid $=104$ Accessed in November 2005

30. Fontoura,M. ,Pree,W. Rumpe, B. The UML profile for framework architectures, AddisonWesley, 2001

31. XMI Documentation at http://www.omg.org/technology/documents/formal/xmi.htm accessed in November 2005

32. Kang, K.C., Kim, S., Lee, J., Kim, K., Shin, E., Huh, M., 1998. FORM: A Feature-Oriented Reuse Method with Domain-Specific Reference Architecture. In: Annals of Software Engineering, vol. 5, 143-168, Kluwer Academic Publishers, Dordrecht, Holland

33. Toacy Cavalcante de OLIVEIRA. Uma abordagem sistemática para a instanciação de frameworks orientados a objetos. [Title in english: A systemactic approach to object oriented frameworks intantiation] Ph.D.Thesis. Port. Presentation: 05/10/01 166 p. Advisor: Carlos José Pereira de Lucena.

34. UML Metal Model Documentation at http://www.omg.org/technology/documents/modeling spec catalog.htm\#UML Accessed in November 2005

35. Parnas, D.L., On the Design and Development on Program Families, IEEE Transaction on Software Engineering, vol. SE-2, n. 1, March 1976, 1-9

36. Meyer, B., Eiffel : The Language Prentice Hall; 1st edition ,October 1, 1991

37. JUnit Documentation found at www.junit.org Accessed in November 2005.

38. J. Bayer, O. Flege, P. Knauber, R. Laqua, D. Muthig, K. Schmid, T. Widen, J. DeBaud. "Pulse: a methodology to develop software product lines". In: Symposium on Software Reusability, 1999. pp. $122-131$

39. FILHO, I,. M.. A documentação e a instanciação de frameworks orientados a objetos. [Title in English: Documenting and instantiating object oriented frameworks] Ph.D.Thesis. Port. Presentation: 17/04/02 184 p. Advisor: Carlos José Pereira de Lucena.

40. XSL Specification at http://www.w3.org/Style/XSL/ Accessed in November 2005

41. Gamma, E., Helm, R., Johnson, R., Vlissides, J., Design Patterns, Elements of Reusable ObjectOriented Software, Addison-Wesley Publishing Company, 1995.

42. UMT Documentation at http://umt-qvt.sourceforge.net/docs/UMT documentation v08.pdf Accessed in November 2005

43. Velocity Template engine at http://jakarta.apache.org/velocity/accessed in September 2005

44. Basic MTL Manual at http://modelware.inria.fr/static pages/docs/html.chunked/BasicMTL UserManual/index.html Accessed in January 2006

45. Atkinson et. al., Component-Based Product Line Engineering with the UML, Addison-Wesley, September 2001

46. Penczek, L., Oliveira T.C. , AFR: an Approach to Systematize Aspect-Oriented Framework Reuse, in Second Asian Workshop on AOSD (AOAsia), co-located with ,ACM International Conference on Automated Software Engineering (ASE), Tokyo, Japan, September 18-22 ,2006

\section{Appendix I}

In order to show its expressiveness, RDL is presented in a BNF-like description. 


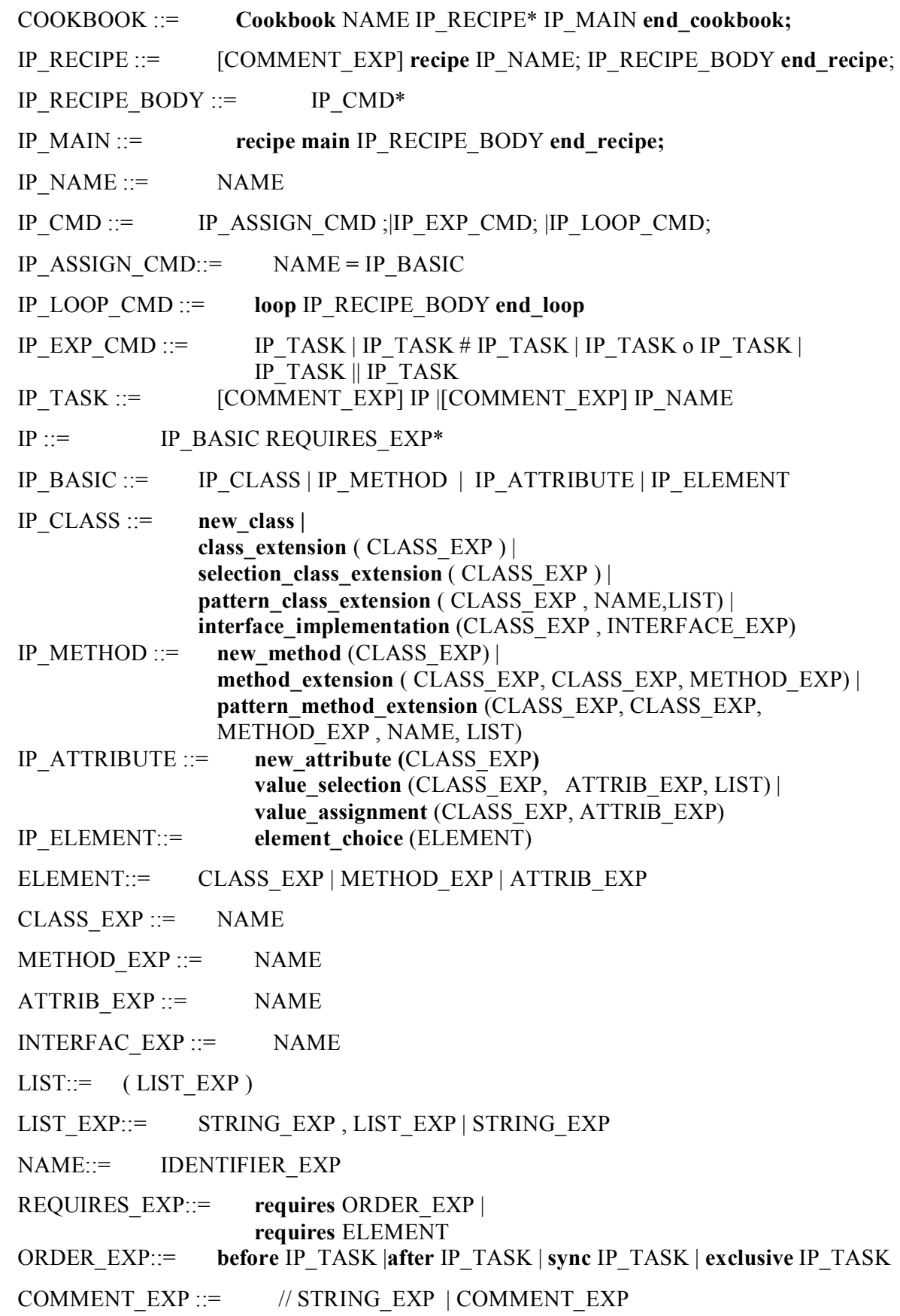


STRING_EXP $::=$ String 